\title{
Finite Element Modeling of Episodic Edifices
}

\author{
Koram Samuel Sakyi ${ }^{1 *}$, Jian-Fei $\mathrm{Lu}^{2}$ \\ ${ }^{1}$ Faculty of Civil Engineering and Mechanics, Jiangsu University, Zhenjiang, China. \\ ${ }^{2}$ Faculty of Civil Engineering and Mechanics, Jiangsu University, Zhenjiang, China. \\ E-mail of corresponding author: samuelkoram@yahoo.com
}

\begin{abstract}
Episodic edifices have a diversity of significant solicitations in contemporary machineries and engineering owing to their exclusive electromagnetic properties. Frequently used episodic edifices comprise; occurrence selective surfaces, visual grilles, phased collection projections, photonic bandgap supplies, and numerous metamaterials. The scrutiny of episodic edifices has all the time been a significant area in computational electromagnetics. This episode, describes a precise and effectual arithmetical study, grounded on a higher-order finite element method (FEM), for depicting the electromagnetic properties of an episodic edifices. Grounded on the Floquet theory, episodic frontier conditions and radioactivity conditions are foremost resultant for the unit cell of an episodic edifice. The FEM is formerly applied to unravel Maxwell's reckonings in the unit cell. To augment the precision and effectiveness of the FEM, rounded elements are employed to discretize the unit cell and higherorder course basis functions are used to enlarge the electrical arena. The asymptotic waveform evaluation (AWE) is applied to implement wild frequency and rawboned curves. To prove the proficiency of the projected FEM, we apply it to the scrutiny of episodic absorbers, incidence selective edifices, and phased collection aerial. For the aerial analysis, a severe waveguide port condition is industrialized to precisely model the aerial feed edifices. In all the occurrences premeditated, acceptable outcomes are obtained.
\end{abstract}

Key words: Episodic, edifice, absorbers, waveguide, electromagnetic.

DOI: $10.7176 /$ CEIS/10-1-04

\section{Introduction}

Episodic edifices have been widely used in electromagnetic engineering. The periodicity in the geometry is habitually subjugated to attain certain desired electromagnetic properties, such as incidence selective performances, which are not attainable in the case of a single component. Numerous heat and visual strategies, such as anechoic compartment absorbers, incidence selective edifices, and phased collection projections, fall into this kind. Arithmetical scrutiny of episodic edifices has been accepted with a diversity of arithmetical approaches, such as the moment technique, the finiteelement method (FEM), and the finite-difference timedomain (FDTD) method. Midst these approaches, the FEM surpasses with demonstrating complex geometry and substantial inhomogeneity. The technique is also adaptable in its capacity to integrate diverse kinds of frontier conditions and diverse excitation approaches deprived of suggestively affecting its structure. The FEM modeling of episodic edifices has been described in prose for mutually scattering [1][5] and radioactivity [6][8] scrutinizes.

This episode, describes a vigorous, higher-order FEM formulation to perfect substantially episodic collection edifices. By means of imposing suitable radioactivity frontier conditions and episodic frontier conditions, the computation area is narrowed to a solitary unit cell in the immeasurable collection. The unit cell inner area is discretized by wavy tetrahedral rudiments for an improved geometry modeling. The electromagnetic arena is then long-drawn-out with higher-order trajectory basis functions, which are more effective than the predictable zeroth-order basis functions. For broadband controls, the asymptotic waveform evaluation (AWE) method can be mutual with the FEM to achieve wild incidence and angular curves. For radioactivity analysis, an exact coaxial feed modeling has been employed, using a severe waveguide port condition. For the tenacity of authentication, arithmetical specimens are given for mutually scattering and radioactivity analyses for several element formations. This statistics and measured outcomes validate the precision and proficiency of the projected FEM formulation.

\section{FEM Formulation}

This section defines several characteristics of the FEM formulation for the scrutiny of episodic edifices. The wild curve methods are also drawn at the conclusion of this section.

\subsection{Discretization}

The edifices under deliberation are planar arrays, infinitely episodic in the $x$ and $y$ orders. Meanwhile all the rudiments in the array are indistinguishable, the FEM analysis is useful only to a single component, or a unit cell, as 
publicized in Fig. 1. Let's commence with Maxwell's reckonings for the electromagnetic arenas inside the calculation field:

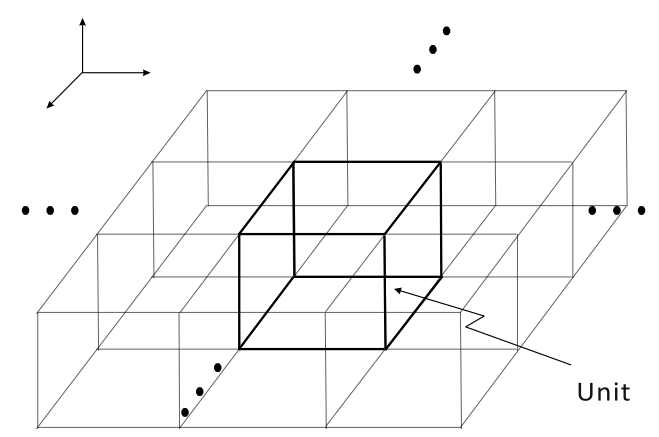

Fig. 1. Unit cell in an immeasurable episodic edifice.

$$
\begin{gathered}
\nabla \times \mathbf{E}=-j \omega \mu_{0} \mu_{r} \mathbf{H} \\
\nabla \times \mathbf{H}=j \omega^{2}{ }_{0}^{2}{ }_{r} \mathbf{E}+\mathbf{J} .
\end{gathered}
$$

Eradicating $\mathbf{H}$ in the two reckonings above results in the upsurge reckoning for $\mathbf{E}$ :

$$
\nabla \times\left(\frac{1}{\mu_{r}} \nabla \times \mathbf{E}\right)-k_{0}^{2} \epsilon_{r} \mathbf{E}=-j k_{0} Z_{o} \mathbf{J} .
$$

Presumptuous that $\mathbf{E}$ placates certain frontier conditions on the exterior $S$ that encircles the calculation area $V$, it can be shown that the unique delinquency is equal to the subsequent variation delinquency

$$
\text { [9]: } \quad \delta F(\mathbf{E})=0
$$

where

$$
\begin{aligned}
F(\mathbf{E})= & \frac{1}{2} \iiint_{V}\left[\frac{1}{\mu_{r}}(\nabla \times \mathbf{E}) \cdot(\nabla \times \mathbf{E})-k_{0}^{2} \epsilon_{r} \mathbf{E} \cdot \mathbf{E}\right] d V \\
& +j k_{0} Z_{0} \iiint_{V} \mathbf{E} \cdot \mathbf{J} d V \\
+ & \text { exterior integral terms }
\end{aligned}
$$

where the exterior integral terms are the outcomes of impressive parallel frontier conditions. This subsection only deliberate on the inner share of the delinquency. The frontier condition terms will be deliberated in the subsequent subsection.

To resolve the variation problem (4) mathematically, the whole calculation area $V$ is discretized into minor rudiments. In the FEM discretization, frequently used rudiments are three-sided rudiments for 2-D glitches and tetrahedral rudiments for 3-D glitches. To authentically perfect curved sides, wavy rudiments are often used. For the wavy rudiments, the assessment of the integrals in (5) is eased by plotting the integrals against a corresponding straight component with unit distance in a new direct system. For three-sided rudiments, the new direct system is signified as the $\xi \eta$ coordinate and the plotting is specified by

$$
\begin{gathered}
x=\sum_{i=1}^{6} N_{i}^{e}(\xi, \eta) x_{i} \\
y=\sum_{i=1}^{6} N_{i}^{e}(\xi, \eta) y_{i} \\
d x d y=|J| d \xi d \eta \quad[J]=\left[\begin{array}{l}
\frac{\partial x}{\partial \xi} \frac{\partial y}{\partial \xi} \\
\frac{\partial x}{\partial \eta} \frac{\partial y}{\partial \eta}
\end{array}\right]
\end{gathered}
$$

where $x_{i}$ and $y_{i}$ are the match up of the $i$ th node in the $x y$ match up system and $N_{i}^{e}(\xi, \eta)$ are scalar form functions demarcated in the $\xi \eta$ match up system. The plotting for tetrahedral rudiments can be demarcated in an alike approach with 


$$
\begin{aligned}
& x=\sum_{i=1}^{10} N_{i}^{e}(\xi, \eta, \zeta) x_{i} \\
& y=\sum_{i=1}^{10} N_{i}^{e}(\xi, \eta, \zeta) y_{i} \\
& z=\sum_{i=1}^{10} N_{i}^{e}(\xi, \eta, \zeta) z_{i}
\end{aligned}
$$

With the mapping

$$
[J]=\left[\begin{array}{lll}
\frac{\partial x}{\partial \xi} & \frac{\partial y}{\partial \xi} & \frac{\partial z}{\partial \xi} \\
\frac{\partial x}{\partial \eta} & \frac{\partial y}{\partial \eta} & \frac{\partial z}{\partial \eta} \\
\frac{\partial x}{\partial \zeta} & \frac{\partial y}{\partial \zeta} & \frac{\partial z}{\partial \zeta}
\end{array}\right]
$$
executed in the novel direct system, where it can be appraised effortlessly using Gaussian Legendre quadrature. It is likewise extra expedient to outline all the basis functions in the novel match up system.

The subsequent phase encompasses intensifying the electromagnetic arenas in rapports of basis functions in respective component. In the framework of 3-D glitches, it is extra expedient to use trajectory or vector basis functions as an alternative to scalar basis functions. For tetrahedral rudiments, the trajectory basis function allied with the $i$ th edge is assumed to be

$$
\mathbf{W}^{e}=\left(L_{i_{1}}^{e} \nabla L_{i_{2}}^{e}-L_{i_{2}}^{e} \nabla L_{i_{1}}^{e}\right) l_{i}^{e}
$$

where $i_{1}$ and $i_{2}$ denote the two vertices associated with the $i$ th edge and $l_{i}^{e}$ is the length of the $i$ th edge. Further, $L_{k}^{e}(k$ $=1,2,3,4)$ are the nodebased basis functions associated with the four vertices of the tetrahedral. It can be demonstrated easily that $\mathbf{W}_{i}{ }^{e}$ automatically satisfies the divergence condition. Thus, the solutions obtained by using vector basis functions are free of spurious solutions. Also, it is easier to model dielectric interfaces and conducting edges and tips using vector basis functions than using scalar basis functions.

The trajectory basis functions demarcated in (13) are zeroth-order inserting functions. Higher-order basis functions can be made methodically by enlarging the zeroth-order inserting functions with Lagrange interpolatory polynomials [10] specified by

$$
\mathbf{N}_{i j k l}^{i_{1} i_{2}}=\gamma \hat{P}_{i}^{p+2}\left(\xi_{1}\right) \hat{P}_{j}^{p+2}\left(\xi_{2}\right) \hat{P}_{k}^{p+2}\left(\xi_{3}\right) \hat{P}_{l}^{p+2}\left(\xi_{4}\right) \mathbf{W}_{i_{1} i_{2}}
$$

(14) where $\left(i_{1}, i_{2}\right)$ are the

amalgamations of two numbers from $(1,2,3,4)$ and $i_{3}$ and $i_{4}$ are two numbers among $(1,2,3,4)$ other than $i_{1}$ and $i_{2}$. Also, $i, j, k$, and $l$ placate with $i+j+k+l=p+2$ where $p$ is the edict of basis functions, $\gamma$ is a standardization constant, $\mathbf{W}_{i 1 i 2}$ are zeroth-order basis functions, and $P_{i}^{\wedge}{ }^{p+2}(\xi)$ are the shifted Silvester polynomials demarcated by

$$
\hat{P}_{i}^{p+2}(\xi)=\frac{1}{(i-1) !} \prod_{l=1}^{i-1}[(p+2) \xi-l]
$$

The thorough discussion of higher-order basis functions can be initiated in [10]. It has extensively been recognized that higher-order basis functions are extra precise and effective in displaying the genuine arena resolution for electromagnetic glitches. By way of an illustration, Fig. 2 displays the comparative blunder against the amount of overall nonentities for using diverse demand basis functions in a 2-D eigenvalue problem. The eigenvalue delinquency is to regulate the cutoff wavenumber of the $T E_{22}$ approach disseminating in a foursided waveguide. The comparative blunder is achieved by likening the arithmetical and logical cutoff wavenumbers. It is effortlessly gotten from Fig. 2 that for a specified integer of nonentities, the higher-order basis functions generate an advanced precision than with the lowerorder basis functions. Furthermore, diverse blunder curvatures in Fig. 2 display diverse grades, signifying that the comparative blunder declines much quicker by picking higher-order basis functions. In an overall sense, one can continuously attain an advanced precision by using higher-order basis functions. Nevertheless, the choice of inserting edict is hinge on explicit glitches. It is prudent and worthwhile to take the edict of basis functions such that it is not much advanced than the edict of geometrical makeover. 


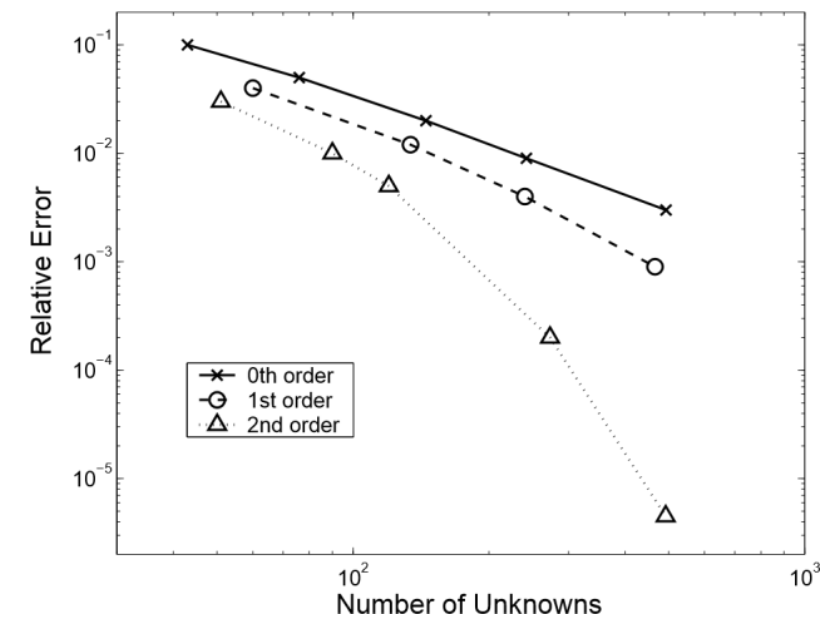

Fig. 2. Comparative blunder in calculating $T E_{22}$ cutoff wavenumer of a $3 \times 2 \mathrm{~cm}$ four-sided (rectangular) waveguide.

As soon as the basis functions are determined, the electronic (or compelling) arena inside each component can be long-drawn-out as

$$
\mathbf{E}^{e}=\sum \mathbf{N}_{i}^{e} E_{i}^{e} \quad{ }_{i=1}^{n}
$$

where $n$ is the integer of inserting points (contingent with the edict of basis functions) inside each component. Replacing (16) into (5), one attains the rudimentary matrices $\left[K^{e}\right]$ per the entries given by

$$
K_{i j}^{e}=\iiint_{V}\left[\frac{1}{\mu_{r}}\left(\nabla \times \mathbf{N}_{i}^{e}\right) \cdot\left(\nabla \times \mathbf{N}_{j}^{e}\right)-k_{0}^{2} \epsilon_{r} \mathbf{N}_{i}^{e} \cdot \mathbf{N}_{j}^{e}\right] d V \quad \text { then }
$$

the right-hand-side (RHS) known vector $\left\{b^{e}\right\}$ specified by

$$
b_{i}^{e}=-j k_{0} Z_{0} \iiint_{V} \mathbf{N}_{i}^{e} \cdot \mathbf{J} d V .
$$

The $\left[K^{e}\right]$ and $\left\{b^{e}\right\}$ are then accumulated to the scheme matrix $[K]$ and scheme known vector $\{b\}$, correspondingly, bestowing to the connectivity of the FEM network (or mesh).

The outcome of subdomain discretization, basis function increase, and finite element accumulating is a set of lined reckonings in the matrix system of

$$
[K]\{E\}=\{b\} .
$$

In cooperation with direct and iterative approaches can be used to unravel the lined scheme reckonings. Prevalent iterative approaches comprise the conjugate gradient (CG) technique and the comprehensive negligible remaining approaches. For direct approaches, the anterior and multi-frontal procedures [11] are appropriate solvers for lined systems subsequent with the finite element accumulating method. In the finite element accumulating method, a huge numeral of rudimentary reckonings are decoupled. Captivating with this fact, the anterior technique does Gaussian elimination as soon as a calculation is totally decoupled as of the rest of the reckonings. The multi-frontal technique is an extension of the anterior system, which makes use of manifold anterior media. The benefit of the anterior/multifrontal technique is its ability to pact with random sparsity form, making it far extra effective than the normal $L U$ decomposition technique. A prevalent multifrontal compendium is called UMFPACK ${ }^{1}$, which is a thin lined solver grounded on the unsymmetric-pattern multi-frontal technique.

\subsection{Frontier Conditions}

Cogitate the unit cell in an immeasurable episodic edifice, as displayed in Fig. 3. The inner capacity, indicated here as $V$, is bounded by four side exteriors, a topmost superficial, and the lowest superficial. It might encompass random dielectric and leading edifices. The four side exteriors $S_{x 1}, S_{x 2}, S_{y 1}$, and $S_{y 2}$ are placed at $x=0, x=T_{x}, y=0$, and $y=T_{y}$, correspondingly, where $T_{x}$ and $T_{y}$ are episodic distances in the $x$ and $y$ directions. The topmost superficial $S_{t}$ is the boundary amongst free-space and the unit cell area. The lowest superficial $S_{b}$ is habitually a ground plane. It might likewise encompass waveguide openings $S_{w}$ that offer excitation for the radioactivity case. In such an overall formation, four classes of frontier conditions are elaborated. On the four side exteriors, episodic frontier conditions are enforced, 
connecting the arenas on the opposite lateral exteriors. On the highest superficial, an episodic radioactivity frontier condition is enacted, which mimics the energy in the direction of the free space in the existence of an immeasurable collection. If a waveguide is present in the structure, then a waveguide port condition is levied on $S_{w}$. Lastly, on conducting exteriors, a perfectly electrically conducting (PEC) frontier condition is imposed explicitly as a uniform Dirichlet frontier condition.

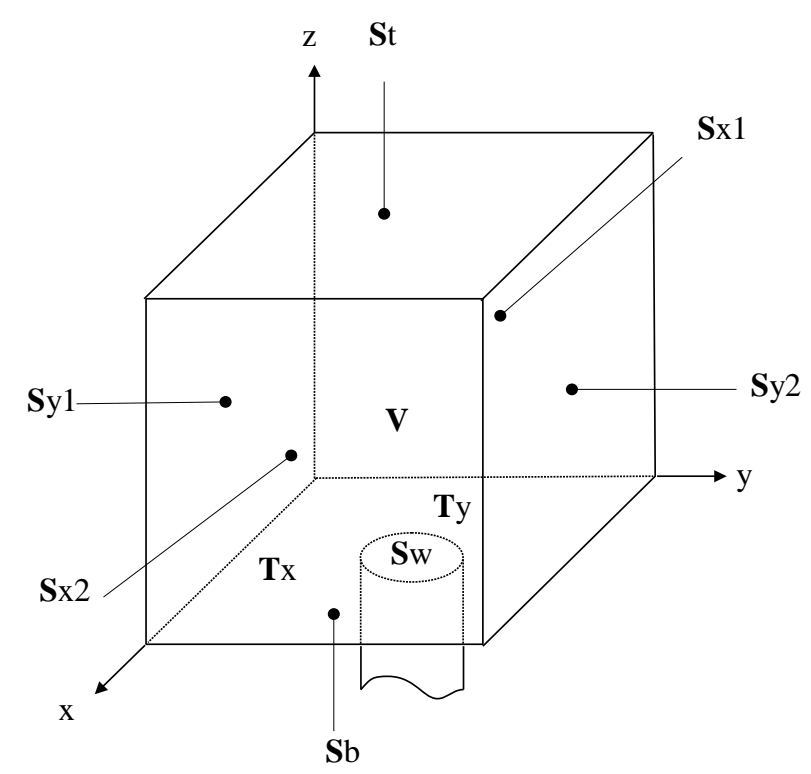

Fig. 3. Diagram of frontiers on a unit cell.

Episodic frontier conditions can be derivate directly from the Floquet theorem. In harmony with the Floquet theorem, the electromagnetic arenas inside and beyond the episodic media placate:

$$
\mathbf{E}\left(x+m T_{x, y}+n T_{y}\right)=\mathbf{E}(x, y) e_{j}\left(m k_{x i} T_{x}+n k_{y i} T_{y}\right)
$$

$\mathbf{H}\left(x+m T_{x, y}+n T_{y}\right)=\mathbf{H}(x, y) e_{j}\left(m k_{x i} T_{x}+n k_{y i} T_{y}\right)$ where 


$$
\begin{aligned}
& k_{x i}=k_{0} \sin \theta_{i} \cos \varphi_{i} \\
& k_{y i}=k_{0} \sin \theta_{i} \sin \varphi_{i}
\end{aligned}
$$

where $\left(\theta^{i}, \varphi^{i}\right)$ are the Episode or image viewpoints.

To ease the application of episodic frontier conditions, undistinguishable exterior interlocks are formed on the opposite lateral exteriors. Formerly for each unidentified $E_{i}$ on one lateral exterior, an equivalent unidentified $E_{j}$ is known on the opposite exterior which has an equivalent comparative point as $E_{i}$. Implementing the Floquet theorem (19), one can simply get the subsequent rapport among $E_{i}$ and $E_{j}$ :

$$
E_{j}=E_{i} e_{j \Psi_{i j}}
$$

where $\Psi_{i j}$ is a point change period specified by

$$
k_{x i} T_{x} \quad \forall E_{i} \in S_{x 1}, E_{j} \in S_{x 2}
$$

$\Psi_{i j}=k_{k y i x i} T T_{y x}+k_{y i} T_{y} \forall \forall E E_{i i} \in \in S S_{y x 11}, E \cap S_{j y 1} \in, E S_{y j 2} \in S_{x 2} \cap S_{y 2 .}(24)$

In the contextual scenery, (23) is an obligatory obviously as an inhomogeneous Dirichlet frontier condition. That is, per each unidentified pair $\left(E_{i}, E_{j}\right): E_{j}$ is removed; the matrix entries allied with $E_{i}$ are changed as

$$
K i l=K i l+K_{j l} e_{j \Psi_{i j}}
$$

for all $E_{l} \in / S_{x 2} \cup S_{y 2}$, then

$$
K_{i k}=K i k+K j l e j\left(\Psi_{i j}-\Psi_{k l)}\right.
$$

for all $E_{l} \in S_{x 2} \cup S_{y 2}$ which is allied with $E_{k}$ by point change $\Psi_{k l}$; and lastly, the RHS trajectory or vector entry allied with $E_{i}$ is changed as

$$
b_{i}=b_{i}+b_{j} e_{j \Psi_{i j}}
$$
by

The functional given in (5) encompasses a superficial integral term over the upper superficial $S_{t}$ by a form specified

$$
S(\mathbf{E})=-j k_{0} Z_{0} \quad \mathbf{M} \cdot \mathbf{H} d S
$$

ZZ

$S_{t}$

where $\mathbf{M}=-n^{\wedge} \times \mathbf{E}$ is the corresponding compelling current on $S_{t}$. Henceforth, there is a requisite to derive a reckoning that narrates the compelling current $\mathbf{M}$ and the magnetic arena $\mathbf{H}$. In order to do that, we foremost assume that a ground plane is placed on $S_{t}$. From the correspondence code, the entire magnetic arena above $S_{t}$ can be inscribed as

$$
\mathbf{H}(\mathbf{r})=\mathbf{H}^{\mathrm{inc}}(\mathbf{r})+\mathbf{H}^{\mathrm{ref}}(\mathbf{r})+\mathbf{H}^{\text {scat }}(\mathbf{r})
$$

where $\mathbf{H}^{\text {inc }}$ is the episode arena and $\mathbf{H}^{\text {ref }}$ is the reflected field by the ground plane. $\mathbf{H}^{\text {scat }}$ is the magnetic field radiated by the magnetic current source on $S_{t}$ in the presence of the ground plane. On invoking the duplicate concept,

$\mathbf{H}^{\text {scat }}$ can be inscribed as

$$
\mathbf{H}^{\mathrm{scat}}(\mathbf{r})=-2 j k_{0} Y_{0} \quad \mathbf{G}^{-}\left(\mathbf{r}, \mathbf{r}^{0}\right) \cdot \mathbf{M}\left(\mathbf{r}^{0}\right) d S^{0}(30) S_{\infty}
$$

where $S_{\infty}$ indicates the immeasurable plane that concurs with the ground plane and $\mathbf{G}^{-}$signifies the dyadic Green's function for free space. Subsequently $\mathbf{M}$ has a spacial episodic property, it can be long-drawn-out in relations to Fourier sequence 


$$
\mathbf{M}^{(x, y)}=\sum_{p, q=-\infty}^{\infty} \tilde{\mathbf{M}}_{p q} e^{-j\left(k_{x p} x+k_{y q} y\right)}
$$

where

$$
\mathbf{M}^{p q}=\begin{gathered}
1 \\
T_{x} T_{y}
\end{gathered} \iint_{S_{t}} \mathbf{M}(x, y) e^{j\left(k_{x p} x+k_{y q} y\right)} d x d y
$$

where

$$
\begin{aligned}
& k_{x p}=\frac{2 \pi}{T_{x}} p+k_{x i} \\
& k_{y q}=\frac{2 \pi}{T_{y}} q+k_{y i}
\end{aligned}
$$

Subsequently, (29) can be written as $\mathbf{H}(x, y)=\mathbf{H}^{\mathrm{inc}}(\mathbf{r})+\mathbf{H}^{\mathrm{ref}}(\mathbf{r})$

$$
-2 j k_{0} Y_{0} \sum_{p, q=-\infty}^{\infty} \tilde{\mathbf{G}}\left(k_{x p}, k_{y q}\right) \cdot \tilde{\mathbf{M}}_{p q} e^{-j\left(k_{x p} x+k_{y q} y\right)}
$$

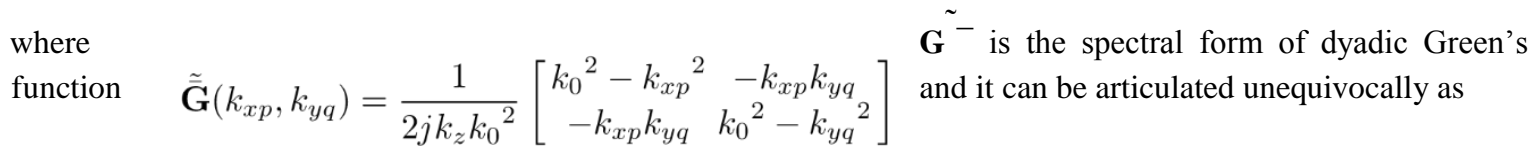
where (36)

$$
k_{z}=\quad k_{02}-k x p 2-k y q 2 .
$$

The integral equation (35) relays the superficial compelling current to its magnetic arena. It implements the radioactivity ailment on the topmost surface of the unit cell in the presence of an immeasurable assortment or array. It is therefore signified as the episodic radioactive frontier condition. By replacing (35) into (28) and then (5), the functional now changes to:

$$
\begin{aligned}
F(\mathbf{E})= & \frac{1}{2} \iiint_{V}\left[\frac{1}{\mu_{r}}(\nabla \times \mathbf{E}) \cdot(\nabla \times \mathbf{E})-k_{0}^{2} \epsilon_{r} \mathbf{E} \cdot \mathbf{E}\right] d V \\
& +j k_{0} Z_{0} \iiint_{V} \mathbf{E} \cdot \mathbf{J} d V \\
& -2 k_{0}^{2} \iint_{S_{t}} \mathbf{M} \cdot \sum_{p, q=-\infty}^{\infty} \tilde{\mathbf{G}}\left(k_{x p}, k_{y q}\right) \cdot \tilde{\mathbf{M}}_{p q} e^{-j\left(k_{x p} x+k_{y q} y\right)} d S \\
& -2 j k_{0} Z_{0} \iint_{S_{t}} \mathbf{M} \cdot \mathbf{H}^{\text {inc }} d S \\
+ & \text { other superficial integral terms. }
\end{aligned}
$$

The discretization of the superficial integral term (28) vintages the compatible matrix entries

$\mathrm{X}_{\infty}$

$$
S_{i j}=-2 k 02 T_{x} T_{y} \mathbf{N}^{\sim} p q i \cdot \mathbf{G}^{\sim-}(k x p, k y q) \cdot \mathbf{N}^{\sim} p q j
$$

where

$\mathbf{N}^{\sim}$ and the RHS known trajectory entries ZZ

$$
b_{i}=-2 j k 0 Z_{0} \quad \mathbf{N} i \cdot \text { Hinc } d S .
$$

where $M$ and $N$ are the maximum are development wavenumbers, which are also denoted as the Floquet harmonics.

By replacing (31) into (30), the integration over the immeasurable exterior is condensed to an infinite synopsis in the spectral field. 
Floquet technique is included in the synopsis. An acceptable integer of Floquet approaches needs to be encompassed to get convergent outcome. On the supplementary side, the arena increase in the FEM defines that the difference in the FEM result cannot surpass the extreme difference that the FEM mesh can decide, which in turn be reliant on the mesh density and the element dictate. This datum ratifies a limit that $M$ and $N$ cannot be quixotically enormous.

Fortunately, numerical tests indicate that the degree of Floquet harmonics drops rapidly with an aggravation $p$ and $q$ and an insertion of seven to nine lowest Floquet harmonics is usually acceptable to yield convergent results.

Meanwhile unequal rudiments are used, the approximation of the integral in (40) needs numerical methods such as the Gaussian-Legendre quadrature. As soon as $S_{t}$ is huge, the estimation of the integrals possibly will take an elongated period.

Accumulating of (39) leads to a lined method that is nonsymmetrical and partially complete, comparatively scarce. Such lined methods can be factorized and resolved professionally by means of the UMFPACK corpus.

To demonstrate the episodic frontier condition and the episodic radiation frontier condition properly model the arena behavior, a simple instance is tested. The test instance consists of a 20-cm-thick even dielectric layer composed of lossy material $\left({ }^{2} r=3-j\right)$ and backed with a ground plane. A plane upsurge is incident from above with episode angle $\theta=60^{\circ}$. The TE and TM control reflection constants, shown in Fig. 4 , are calculated over $0.1 \sim 0.7 \mathrm{GHz}$ frequency band. In the FEM calculation, the unit cell is shown as a $20 \times 20 \times 20 \mathrm{~cm}$ constant dielectric box with a milled plane positioned on its lowest superficial. The virtuous treaty amongst the FEM fallouts and the analytical results validates the correct application of the episodic frontier condition and the episodic radiation frontier condition.

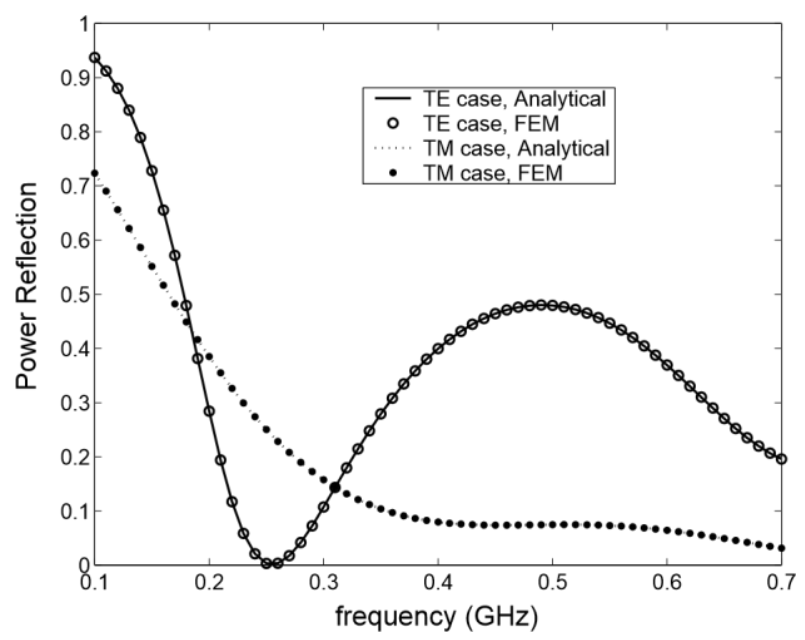

Fig. 4. Power reflection constants for the 20 -cm-thick unvarying layer $\left({ }^{2}=3-j\right)$ supported by a milled plane at $\theta=60^{\circ}$.

In dynamic array edifices, such as array projections, waveguides are often used to feed each projection component. The waveguide well-thought-out here is a uniform waveguide with a random cross section. For hollow waveguides, proliferating means are TE and TM methods. For coaxial lines, an extra TEM method survives. Deprived of losing simplification, the electric arena on the waveguide space $S_{w}$ can be inscribed as a totality of different waveguide methods or modes:

$$
\mathbf{E}(\mathbf{r})=\mathbf{E}^{\text {inc }}(\mathbf{r})+\sum_{m=0}^{\infty} C_{m} \mathbf{e}_{m}(\mathbf{r}) e^{j k_{z m} z}
$$

where

$$
k_{z m}=\overline{k 02-k_{c m 2}} \quad \mathrm{q}
$$

where $k_{c m}$ and $\mathbf{e}_{m}$ are cut-off wavenumbers and transverse means functions allied with the $m$ th means. They can be attained arithmetically by resolving the parallel 2-D eigenvalue problem using the FEM. The means index $m$ comprises of all possible means (TE, TM, and TEM if conceivable), and $\mathbf{E}^{\text {inc }}$ is the occurrence arena. For easiness, we assume central means incidence; that is, $\mathbf{E}^{\text {inc }}=\mathbf{e}_{0}$. The $C_{m}$ is the replication constant for each means. Using orthogonality amongst different methods, $C_{m}$ can be articulated as $\mathrm{ZZ}$

$$
C_{m}=e_{-j k_{z m z}} \quad \mathbf{e} m \cdot(\mathbf{E}-\mathbf{E} \text { inc }) d S .
$$


A frontier form can be derived by replacing (44) into (42), and then applying ${ }^{\wedge} n \times \nabla \times$ operator on mutual sides of the reckoning, yielding

$$
\hat{n} \times \nabla \times \mathbf{E}-j k_{0} Z_{0} \sum_{m} Y_{m} \mathbf{e}_{m} \iint_{S_{w}} \mathbf{e}_{m} \cdot \mathbf{E} d S=-2 j k_{0} Z_{0} Y_{0} \mathbf{e}_{0}
$$

where $Y_{m}$ are modal admittances specified by

$$
\begin{aligned}
& \text { for TE means } \\
& \begin{array}{l}
\text { for TEM means. } \\
\begin{array}{l}
\text { Implementing } \\
\text { practical (5): }
\end{array}
\end{array} \\
& \qquad S(\mathbf{E})=\frac{j k_{0} Z_{0}}{2} \iint_{S_{w}} \mathbf{E} \cdot\left(\sum_{m} Y_{m} \mathbf{e}_{m} \iint_{S_{w}} \mathbf{e}_{m} \cdot \mathbf{E} d S\right)-2 Y_{0} \mathbf{E} \cdot \mathbf{e}_{0} d S \text {. } \\
& \frac{1}{k_{z m} Z_{0}}
\end{aligned}
$$

frontier condition (45) on $S_{w}$ yields an extra superficial integral term in

Once discretization and arena enlargement, (47) can be cast hooked on the subsequent medium setup as

$$
\begin{aligned}
S_{i j} & =j k_{0} Z_{0} \sum_{m} Y_{m} \hat{N}_{i}^{m} \hat{N}_{j}^{m} \\
b_{i} & =2 j k_{0} Z_{0} Y_{0} \hat{N}_{i}^{0}
\end{aligned}
$$

where

$$
\hat{N}_{i}^{m}=\iint_{S_{w}} \mathbf{N}_{i} \cdot \mathbf{e}_{m} d S
$$

Accumulating the above medium or matrix entries outcomes in a complete submatrix in the scheme medium $[K]$, as in the instance of the episodic radiation frontier condition. The calculation of (48) needs the calculation of the integral (50), which is likewise calculated arithmetically. In computer application, the boundless totality in (47) is of a necessity be curtailed. The integer of waveguide approaches involved in (47) ought to be huge enough to symbolize the real arena supply on the waveguide gap. Nonetheless, the disparity of the utmost means involved ought not to surpass the difference that the FEM mesh can receive.

To authenticate the waveguide haven condition, a waveguide joint cutoff delinquency is measured. The waveguide joint comprises of dual units of concentric spherical waveguides, with a range of $1.5 \mathrm{~m}$ and $0.95 \mathrm{~m}$, correspondingly. A $T E_{11}$ upsurge is incident from the greater unit and the control replication constant for the $T E_{11}$ method detected at the greater unit is calculated. In this sample, the waveguide port conditions are obligatory on the waveguide cross sections to shorten the calculation field, and 25 sophisticated means plus the principal method are encompassed in the frontier condition. Figure 5 shows the calculated replication constant, which decides well by the elucidation gotten by the means equivalent technique. The arithmetical sample displays obviously that the waveguide port condition appropriately replicas the arena performance on the boundary of the waveguide intersection.

\subsection{Fast Curve Methods}

The invention obtained in the preceding two subcategories is an incidence field technique, which means that the scheme matrix has to be made and resolved for each precise occurrence. In numerous of real-world applications, broadband frequency responses must to be calculated. One might conceive that the overall time required for such frequency curves will develop undesirably

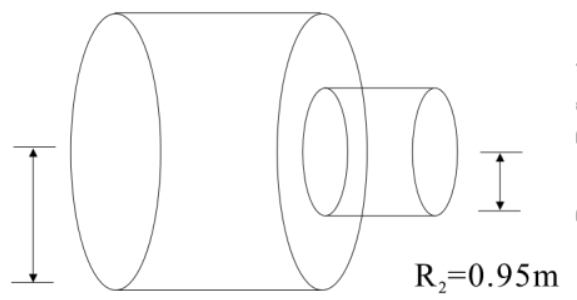

$\mathrm{R}_{\mathrm{1}}=1.5 \mathrm{~m}$

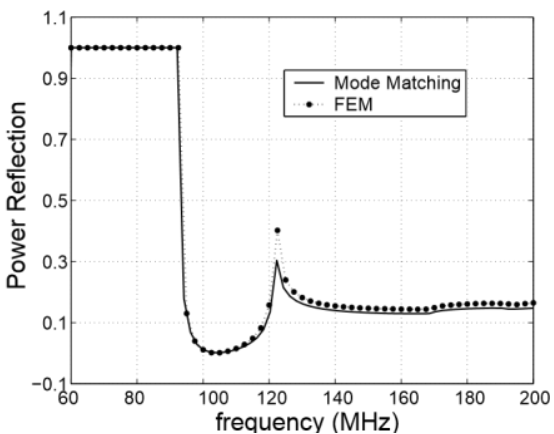

(b) 
Fig. 5. (a) Geometry of a spherical waveguide joint. (b) Power replication constant for $T E_{11}$ approach.

long. In this subcategory, the AWE technique is mutual with the FEM design to execute wild curves. The AWE method was initially presented in circuits' exploration [12]. Its solicitations in the frequency-domain FEM and MoM have been presented lately [13]-[15].

In the framework of electromagnetic glitches, the lined scheme of reckonings to be resolved is

$$
[A]\{x\}=\{b\}
$$

where mutually the matrix and the trajectories are functions of wavenumber (or frequency). First we want to enlarge $\{x\}$ in relations to Taylor series

$$
\{x\}=\sum_{i=1}^{n} \frac{\{x\}^{(i)}}{i !}\left(k-k_{0}\right)^{i}
$$

where $k_{0}$ is the enlargement wavenumber. In order to determine $\{x\}^{(i)}, i=$

$1,2, \cdots, n$, one can take the $n$th derivative of the mutual verges of (51) to get

$$
([A]\{x\})^{(n)}=\{b\}^{(n)} \text {. }
$$

The left-hand side of the above calculation can be simply long-drawn-out as

$$
\sum_{i=0}^{n} \frac{n !}{i !(n-i) !}[A]^{(i)}\{x\}^{(n-i)}=\{b\}^{(n)}
$$

As of (54), a recursive formulation can be derived to analyze $\{x\}^{(n)}$, which is given by

$$
\{x\}^{(n)}=[A]^{-1}\left(\{b\}^{(n)}-\sum_{i=1}^{n} \frac{n !}{i !(n-i) !}[A]^{(i)}\{x\}^{(n-i)}\right) .
$$

After the Taylor constants are gotten, the Taylor sequence is then rehabilitated to the conforming Pad'e sequence

$$
\{x\}=\frac{\sum_{i=0}^{L}\{a\}_{i}\left(k-k_{0}\right)^{i}}{1+\sum_{j=1}^{M}\{b\}_{j}\left(k-k_{0}\right)^{j}} .
$$

The Pad'e enlargement is extra proficient of apprehending the poles of the transmission function and consequently yields a greater conjunction range. In instances where the conjunction series of one particular enlargement point is not adequate to offer a precise answer over the whole incidence band, a manifold point enlargement method named complex frequency hopping $(\mathrm{CFH})$ can be used [13]. In $\mathrm{CFH}$, a binary pursuit procedure is used to regulate the enlargement points adaptively pending the whole frequency band is covered by the over-all merging zones of the enlargement points. The procedure is delineated as follows. Assumed a curve array $\left[k_{\min }, k_{\max }\right]$ and an error tolerance $\varepsilon$ :

(1) Apply AWE at $k_{\min }$ and $k_{\max }$; get the conforming enlargement functions $x_{1}(k)$ and $x_{2}(k)$.

(2) Let $k_{\operatorname{mid}}=\left(k_{\min }+k_{\max }\right) / 2$ and estimate $x_{1}\left(k_{\operatorname{mid}}\right)$ and $x_{2}\left(k_{\operatorname{mid}}\right)$.

(3) If $\left|x_{1}\left(k_{\text {mid }}\right)-x_{2}\left(k_{\text {mid }}\right)\right|<\varepsilon$, the procedure halts; else, the above phases are frequent for sub-regions $\left[k_{\min }, k_{\text {mid }}\right]$ and $\left[k \operatorname{mid}, k_{\max }\right]$.

In overall, the wild curve method described above is considerably extra effective in creating broadband occurrence responses likened to the point by-point method. Figure 6 displays the image constant for a 1-D episodic dielectric edifice above occurrence band 130 176 MHz. The AWE solver provides similar outcome as the direct cunning at respective occurrence point. Table 1 displays the contrast of calculation period for using the direct and AWE solvers. It is understood from the table that the over-all calculation period essential by the AWE solver is considerably fewer than that required by the direct solver.

The conjunction range of an enlargement point is mostly resolute by two features: the maximum order of the derivatives and the exactness of the derivatives. As of the application of the episodic frontier condition and the episodic radiation frontier condition, the derivatives of matrix entries with reverence to wavenumber usually cannot be considered logically in closed systems, which is diverse from [16] where logical derivatives are available. Consequently, most derivatives are considered arithmetically. This levies boundaries on both the uppermost order and the exactness of the derivatives. 


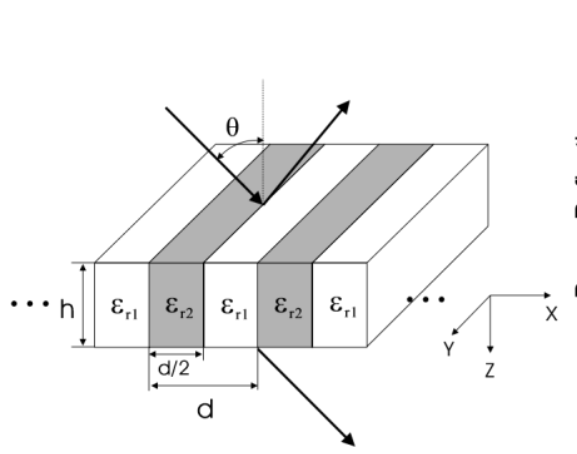

(a)

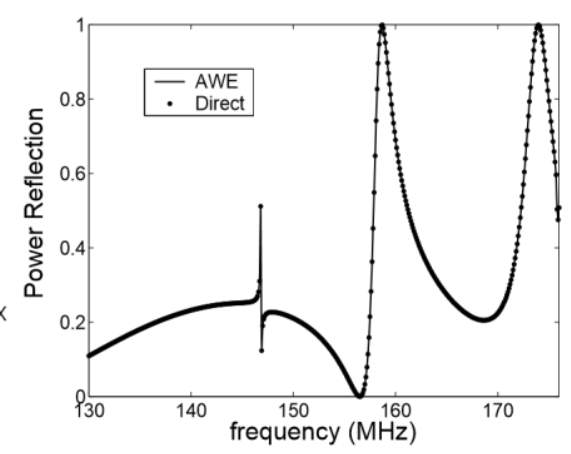

(b)

Fig. 6. (a) Geometry of the dielectric sheet with intermittently fluctuating permittivity with ${ }^{2}{ }_{r 1}=1.44,{ }^{2}{ }_{r 2}=2.56, d=1 \mathrm{~m}, h=2.037 \mathrm{~m}, \theta$ $=45^{\circ}$. (b) Specular power replication constant.

Table 1. Contrast of calculation period for occurrence sweep.

\begin{tabular}{|c|c|c|c|}
\hline $\begin{array}{c}\text { Solver } \\
\text { type }\end{array}$ & $\begin{array}{c}\text { Period for a single } \\
\text { frequency point (s) }\end{array}$ & Number of points & $\begin{array}{c}\text { Total period } \\
(\mathrm{s})\end{array}$ \\
\hline Direct & 53.2 & 461 & 24525 \\
\hline AWE & 116.7 & 6 & 700.2 \\
\hline
\end{tabular}

Several significant constraints of array edifices, such as image design, are angular reactions. It is simply understood that the structure matrix entries are reliant on event viewpoints (or image viewpoints) as an outcome of the enforcement of the episodic frontier condition (23) and the episodic radioactivity condition (35). This is diverse from the non-periodic instance, in which only the RHS identified trajectory is angle-dependent and the structure matrix is factorized only once for all viewpoints of frequency. Nevertheless, for episodic edifices, the structure matrix is of a necessity to be renewed and refactorized at each event viewpoint.

To quicken wide-ranged angular curves, the AWE method defined above for incidence curve can be expressed for angular curve in a much related way. In most image scrutinizes, $\theta$ is the image viewpoint. It is consequently expedient to describe an angle-dependent wavenumber $k_{t}=k_{0} \cos \theta$. Before each amount in (51) can be written as a function of $k_{t}$ and its derivatives with respect to $k_{t}$ can be calculated. Succeeding the same processes as defined in this subdivision, one will lastly get a set of enlargement constants at numerous enlargement points $k_{t 1}, k_{t 2}, \cdots, k_{t n}$, from which the anticipated amount over the whole angular array can be deduced.

Figure 7 displays the angular curve of the specular replication constant

for the similar instance as displayed in Fig. 6 at $f=140 \mathrm{MHz}$. The two curvatures calculated by the AWE solver and the direct solver overlay upon each other. Table 2 displays the contrast of calculation period for scheming the whole angular response. It is obvious that the AWE solver outpaces the direct solver in relations of overall calculation period.

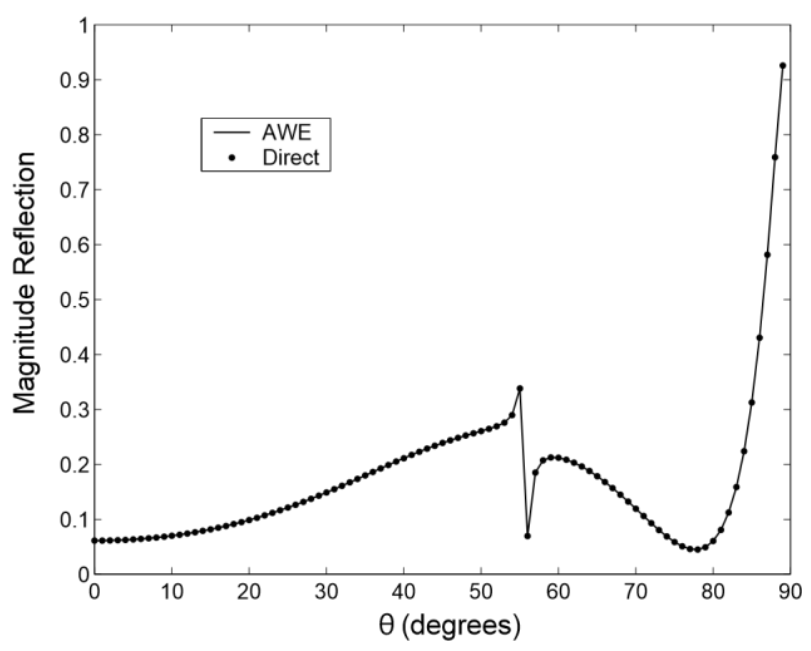

Fig. 7. Specular degree replication constant versus occurrence angle for the dielectric sheet in Fig. 6 at $f=140 \mathrm{MHz}$. 


\begin{tabular}{|c|c|c|c|}
\hline $\begin{array}{c}\text { Solver } \\
\text { type }\end{array}$ & $\begin{array}{c}\text { Time for a single } \\
\text { frequency point (s) }\end{array}$ & Number of points & Total time (s) \\
\hline Direct & 53.2 & 90 & 4788 \\
\hline AWE & 116.7 & 8 & 933.6 \\
\hline
\end{tabular}

\section{Arithmetical Outcomes}

K. Samuel S. (2018), posit that this subdivision presents instances of FEM analysis of episodic edifices including episodic absorbers, occurrence selective edifices, and phased range projections. The arithmetical instances authenticate the FEM design drawn in Section 2 and prove its competences.

\subsection{Episodic Absorbers}

Episodic absorbers are comprehensively used as low-reflection layer of the metal protecting fortifications in electromagnetic compatibility capacities. Scattering analysis of episodic absorbers originally resorted to logical approaches. At exact high incidences, asymptotic approaches such as the constant model of deflection [17] can be useful. At exact low incidences, the unique problem can be converted into a multilayer problem and estimated results can be gotten [18], [19]. Current improvements in full-wave arithmetical approaches allow an extra universal and extra precise scrutiny. Episodic form of the MoM has been expressed and extensively used [20]-[23]. There likewise exists incidenceand time-domain finite change displaying of episodic fascinating edifices [24]-[26]. The FEM is best appropriate for displaying complex absorber geometry and configuration and has remained used in the study and design of episodic absorbers [5], [27].

Figure 8 displays the component cell in an episodic absorber range. The fascinating material is characteristically molded into segments for 2-D ranges and pyramid-cones for 3-D ranges. In the FEM analysis, the component cell covers only one single absorber. On the four side exteriors of the component cell, the episodic frontier conditions (23) are levied. The highest (or top) superficial $S_{t}$ is located somewhere above the tip of the absorber and the episodic radiation frontier condition (35) is levied there. The lowest (or bottommost) surface $S_{b}$ is located on the corporeal frontier of the absorber, which is habitually a PEC pulverized plane that functions as an electromagnetic defense. Afterwards the electrical arena is resolved ubiquitously inside the component cell, the specular replication constant $R(p=0, q=0)$ can be calculated from the opening arenas on the highest superficial $S_{t}$, giving

$$
\begin{aligned}
& R=\mathbf{H r e f}_{t} \mathbf{H}+\text { inc }_{t} \mathbf{H}_{\text {scat }_{t}} \\
& =1-2 j k_{0} Y_{0} \tilde{\mathbf{G}^{-}}\left(k_{x 0}, k_{y 0}\right) \cdot \mathbf{M}_{00}^{\sim}
\end{aligned}
$$

where $Y_{0}=1 / Z_{0}$ and $\mathbf{M}_{p q}^{\sim}$ and $\tilde{\mathbf{G}^{-}}\left(k_{x p}, k_{y q}\right)$ are well-defined in (32) and (36), correspondingly.

First and foremost, we scrutinize the immersion features of a metal-backed planar stratified absorber, which has been scrutinized with the superficial fundamental reckoning technique [22]. The absorber is made up of three immeasurable sheets, each with a separate substantial property. The geometry is displayed in the insertion of Fig. 9. For contrast, the physical properties of the three sheets have been selected to be like those in [22]. The intended specular replication constants from 2 to $18 \mathrm{GHz}$ are conspired in Fig. 9. For this situation, the

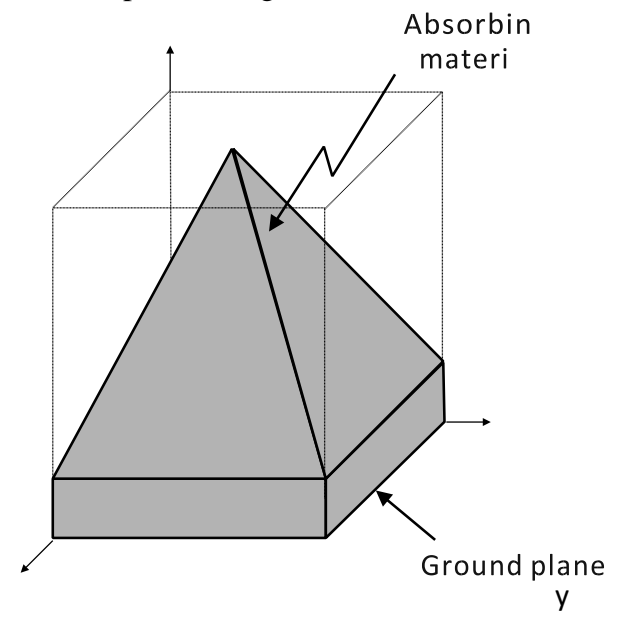


Fig. 8. Component cell in an episodic absorber array.

analytic resolution is gotten by surging the transmitter matrix for every sheet. It is perceived that the arithmetical outcome approves well with the diagnostic result.

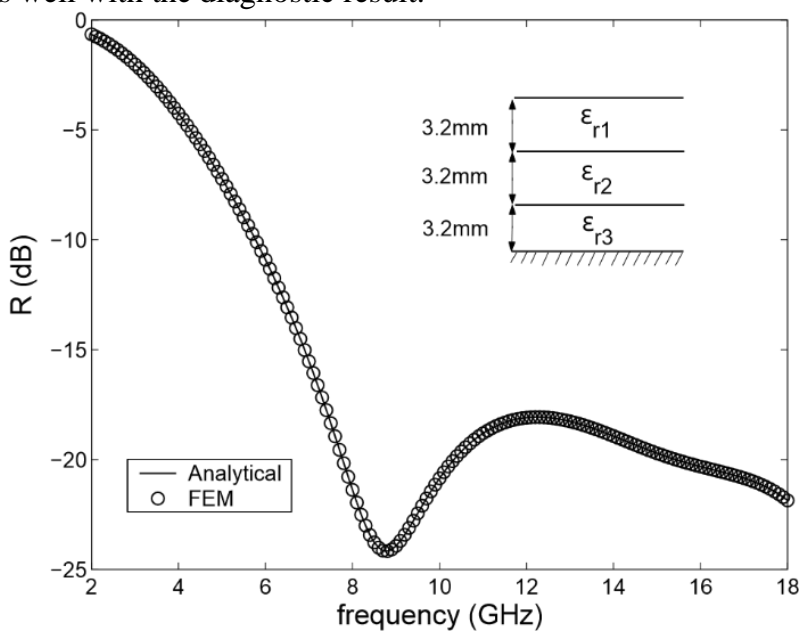

Fig. 9. Specular power replication for a stratified absorber at regular frequency.

To assess the concerts of higher-order trajectory components, we review the stratified absorber sample by assessing the root-mean-square (RMS) blunders for using diverse guidelines. To liken diverse guidelines, two dissimilar mesh magnitudes are used and for each of them the RMS blunders are calculated using zeroth-, first, and second-order tetrahedral components, respectively. The RMS blunders as opposed to the number of nonentities are displayed in Fig. 10. In the calculation, the incidence is permanent to be $30 \mathrm{GHz}$ and the replication constants are calculated as opposed to episode viewpoints. The RMS blunder is demarcated as

$$
\mathrm{RMS}=\sqrt{\frac{1}{N_{s}} \sum_{i=1}^{N_{s}}\left|R_{i}-R_{a}\right|^{2}}
$$

where $R_{a}$ and $R_{i}$ are the diagnostic and calculated replication constants, respectively, and $N_{s}$ is the sum of episode viewpoints. It is evident from Fig. 10 that the exactness advances with accumulative component order. It is also well-known that, for a similar number of nonentities, more precise outcomes can be gotten by using higher-order basics. This instance proves the benefit of using higher-order FEM.

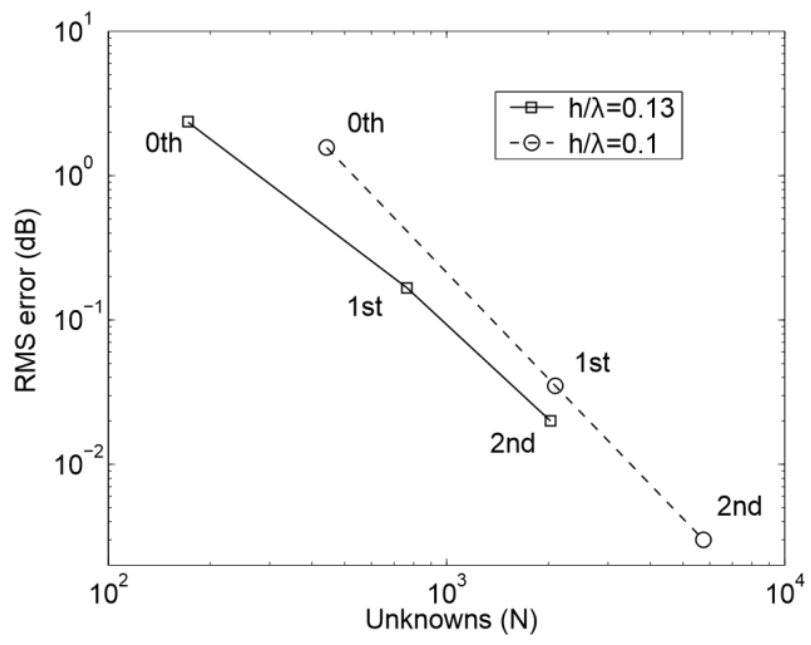

Fig. 10. Evaluation of RMS blunders for dissimilar mesh magnitudes and component orders.

Additionally, we scrutinize the replication from a wedge absorber as opposed to episode viewpoints. The absorber is singly episodic in the $x$ path and constant in the $y$ path, and has a comparative permittivity ${ }_{r}=1.45-0.4 j$. The 
comprehensive geometry is presented in the insertion of Fig. 11(a). The plane of frequency is expected to be the $x z$ plane. Figures 11(a) and (b) liken the outcomes by the FEM encryption and those by a low-frequency calculation technique (or the supposed homogenization technique) for $f=100$ and $300 \mathrm{MHz}$, correspondingly. In the low-slung incidence calculation, the absorber is substituted with more than a few similar layers, whose active permittivity and porousness are resolute from the outline and quantifiable configuration of the novel absorber. The estimate is effective as long as the era of the absorber is trivial likened to the wavelength. The contrasts display that at minor incidences the two approaches forecast a comparable replication form, whereas at sophisticated incidences some differences are detected. In the final case, the period is to some extent bigger than one half of the wavelength. In this instance, the absorber is not supported by a steering plane.

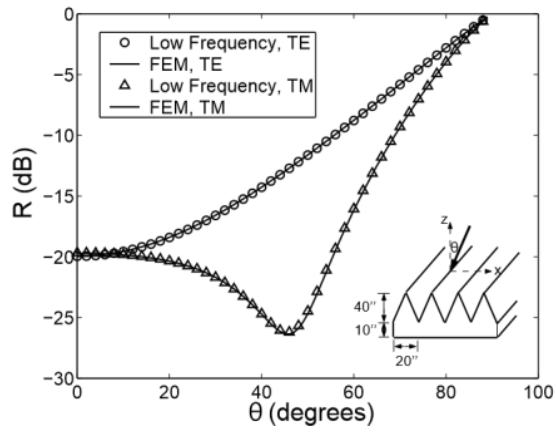

(a)

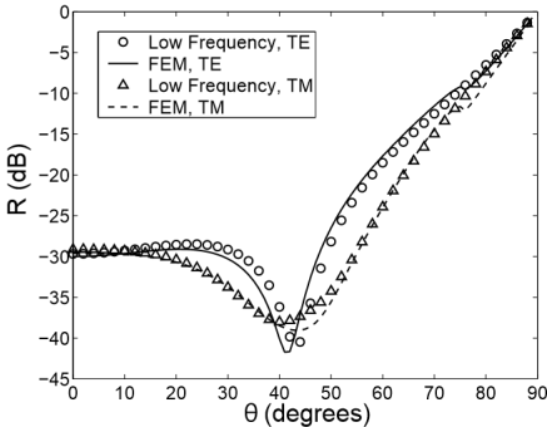

(b)

Fig. 11. Specular power replication for the segment absorber with ${ }^{2}=1.45-0.4 j$. (a) $f=100 \mathrm{MHz}$. (b) $f=300 \mathrm{MHz}$.

Subsequently, we present some instances of extra episodic absorbers. Figure 12(b) displays the specular power replication constant for an extra episodic absorber selection whose component cell geometry is displayed in the insert. In this instance, the permittivity of the fascinating material is a function of incidence, as shown in Fig. 12(a). To implement the fast frequency sweep, it is more convenient to expand the permittivity as a set of known functions of frequency, such as polynomial functions

$$
\epsilon(f)=\sum_{n=0}^{N} a_{n}\left(1-\frac{f}{f_{\max }}\right)^{n}
$$

where $a_{n}$ are the enlargement constants resolute from the material's permittivity features and $f_{\max }$ is the extreme comprehensive incidence. Typically, $N=4$ is adequate for an upright molding of genuine permittivity. The specular replication constant is intended for incidences amid 30 and $200 \mathrm{MHz}$. Outcomes from three diverse approaches are likened: the FEM, the finite-difference frequency-domain (FDFD) technique [24], and the low incidence calculation technique. It is perceived that the three approaches come to an agreement with each other for minor incidences and fluctuate by about $2 \mathrm{~dB}$ at advanced incidences. As extra instance, Fig. 13(b) displays the geometry and calculated specular replication for an extra episodic absorber of related pyramid form. The frequency-dependent permittivity used in this calculation is specified in Fig. 13(a). The FEM outcome is likened to the low-frequency calculation and with the outcomes by Jiang and Martin [5] for the $50 \mathrm{MHz}$ to $1 \mathrm{GHz}$ incidence array. Moreover at minor incidences, the three outcomes come to an agreement well. The changes at sophisticated incidences possibly will effect owing to the low power level of the replicated arena (less than $-50 \mathrm{~dB}$ ). Likewise the situation for the low-frequency calculation to be effective possibly will not be content at these high incidences.

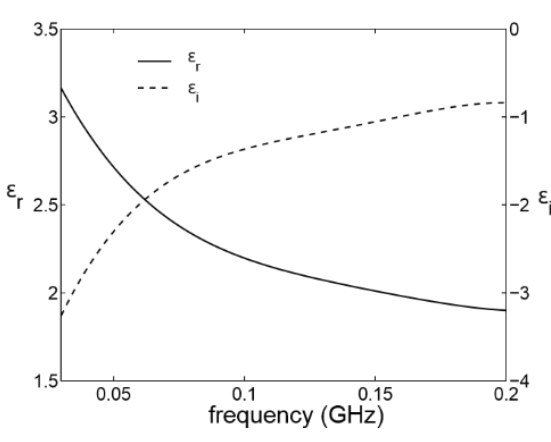

(a)

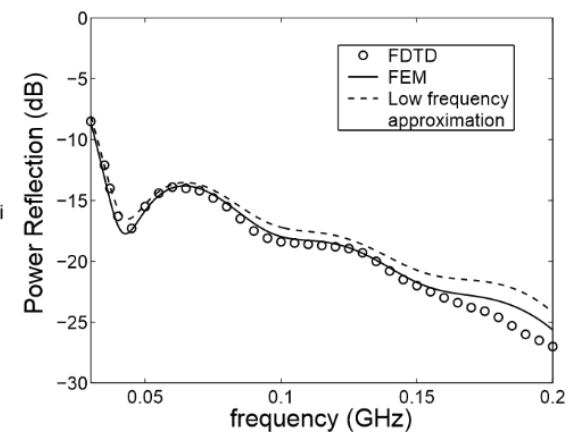

(b) 
Fig. 12. (a) Multifaceted permittivity and (b) specular power replication for a metal-backed pyramid absorber with an era of 24 in and height of 96 in at standard frequency.

As a final point, we examine the consequence of the pyramid shape on the absorber enactment. Figure 14 displays three diverse pyramid shapes that are under deliberation. They altogether have similar altitude and base measurements, but the lateral exteriors of the pyramid are plane, bowl-shaped, and curved, correspondingly. The range of curving $R$ is 70 in for mutually bowl-shaped and curved exteriors. The calculated specular replication is displayed in Fig. 15. The outcomes display that,

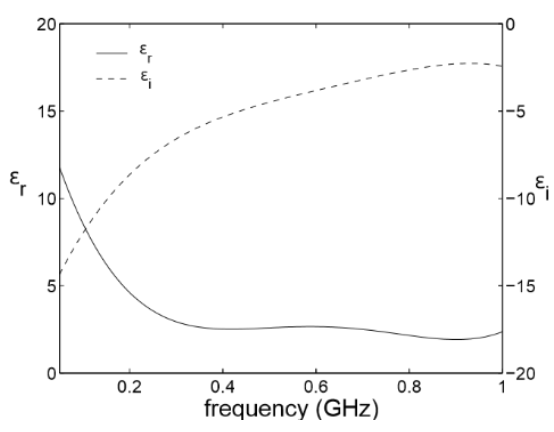

(a)

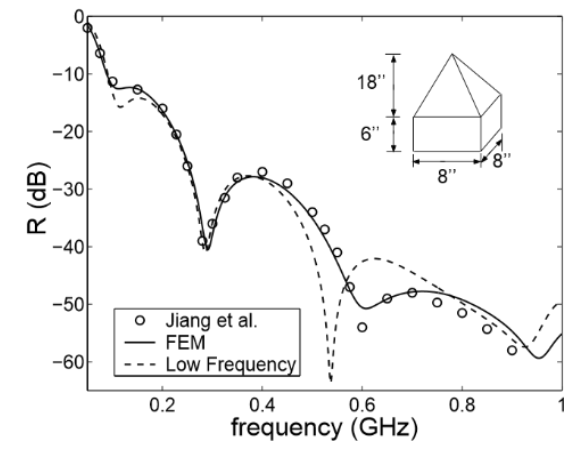

(b)

Fig. 13. (a) Multifaceted permittivity and (b) specular power replication for a metal-backed pyramid absorber with an era of 8 in and altitude of 24 in at standard frequency
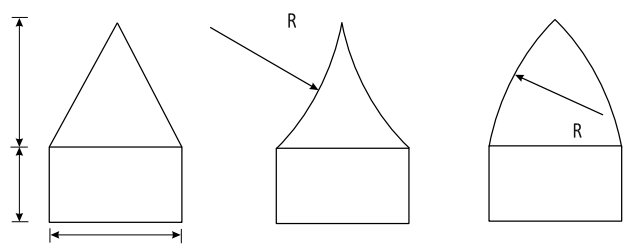

Fig. 14. Lateral view of pyramids with flat, bowl-shaped, and curved lateral exteriors.

apart from at very low incidences, the flat-surface pyramid outpaces both the bowl-shaped- and the curvedsurface pyramids.

\subsection{Incidence Choosy Edifices}

Incidence choosy edifices (FSSs) are 2-D ranges of metal and/or dielectric developments, whose handful reaction is branded by a passband or stopband, at which the overall spread or replication happens. FSSs have been scrutinized using diverse approaches. Diagnostic study of 1-D episodic dielectric sheets was made in [28] grounded on a conducted wave concept. Regarding arithmetical approaches, integral reckoning based approaches, such as the MoM, can be employed [29]-[31]. Time-domain scrutiny of FSSs was conveyed lately using the FDTD technique [32]. Since the FEM can simply perfect unequal figures, it is habitually chosen for extra complex edifices. Effective FEM scrutiny of FSSs has been described in [4] and [33]. Adding up to this, a cross technique that cartels the FEM with the generalized 


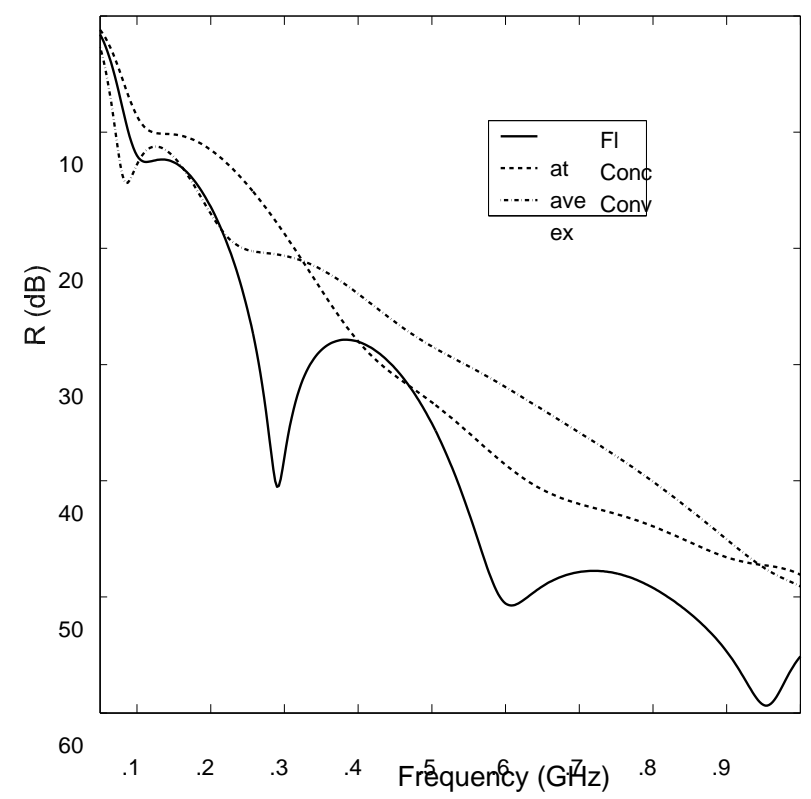

Fig. 15. Specular power replication for metal-backed pyramid absorbers with flat, bowl-shaped, and curved lateral exteriors at standard frequency.

sprinkling matrix (GSM) technique through a surging method was anticipated to exploit efficacy [34].

Figure 16 displays a basic FSS component cell. It possibly will consist of one or more than a few dielectric sheets which contain metal surfaces or edifices. For easiness, the metal exteriors are measured infinitesimally tinny and faultlessly conductive, nonetheless this is not prerequisite in the preparation. In fact, as of the volumetric discretization, the metallic and dielectric can take any form and structure. The component cell is surrounded by four side exteriors and a highest and a lowest superficial. On the four side exteriors, the episodic frontier conditions (23) are levied. On the highest and lowest exteriors, the episodic radiation frontier conditions (35) are imposed with the episode period in (35) omitted for the lowest superficial $S_{b}$. Next the electric arena is resolved for everywhere inside the component cell, the replication constant can be calculated using (64) and the conduction constant can be calculated likewise as

$$
T=\mathbf{H}_{-} \mathbf{H}_{\text {scat }_{t} \text { inc }_{t}}
$$

$$
=-2 j k_{0} Y_{0} \tilde{G}^{-}\left(k_{x 0}, k_{y 0}\right) \cdot \mathbf{M}^{\sim} 00
$$

where $\mathbf{M}^{\sim}$ on is premeditated from (32) with the superficial integration carried out on $S_{b}$.

First and foremost, we consider a 1-D episodic dielectric sheet whose permittivity

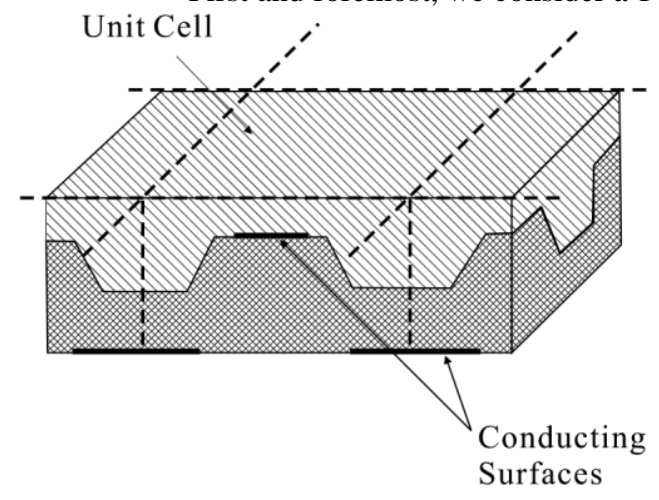

Fig. 16. Obverse view of a basic incidence selective edifice. 
substitutes between ${ }^{2} r 1=1.44$ and ${ }^{2} r 2=2.56$ along the $x$ path, as shown in Fig. 6(a). A TE plane wave is episode from one lateral of the slab at the event viewpoint $\theta=45^{\circ}$. Two instances are calculated, with standardized slab height $h / d$ selected to be 1.713 and 2.037, as stated in [28]. The computed replication constants are displayed in Figs. 17(a) and 17(b). For contrast, the diagnostic results by Bertoni et al. [28] are also plotted. It is displayed that the FEM precisely envisages the diagnostic booming bands in both cases. We also memo that merely five to six enlargement points are used in the wild incidence curve, but all the comprehensive structures have been apprehended.

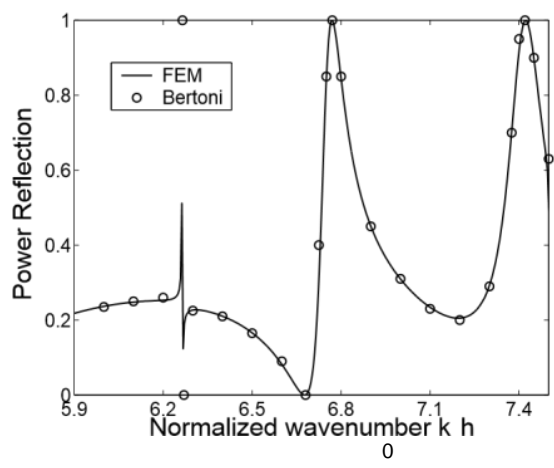

(a)

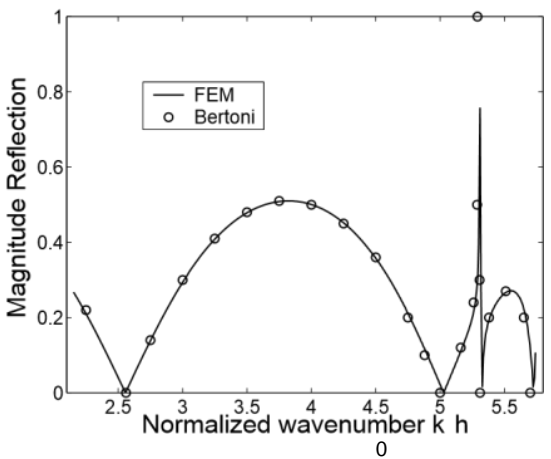

(b)

Fig. 17. (a) Specular magnitude replication constant for $h / d=1.713$. (b) Specular power replication constant for $h / d=2.037$.

The subsequent instance concerns a sphere-shaped slot FSS whose component cell geometry is displayed in Fig. 18(a). Figures 18(b) and 18(c) display the power conduction constants at standard incidence for the free-standing

FSS and the FSS printed on a $0.3-\mu$ m-thick dielectric substrate with permittivity ${ }_{r}=16$, correspondingly. Both outcomes are likened with those by Volakis et al. [4]. Once a rougher web is used in the calculation of Fig. 18(c),

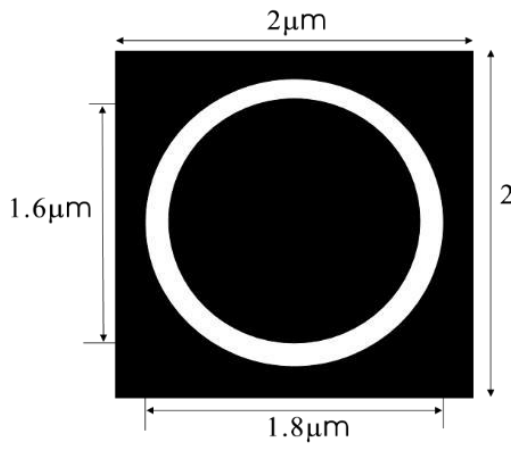

(a) the top of our outcome changes to a lengthier wavelength.

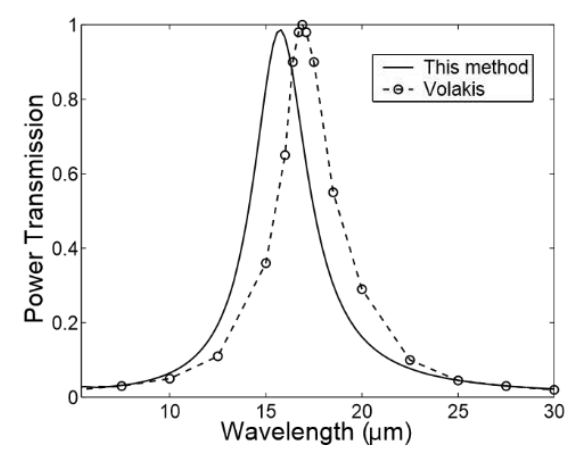

(c) Fig. 18. (a) Highest viewpoint of a spherical slot FSS. (b) Specular power conduction constant for the free-standing FSS. (c) Specular power conduction constant for the FSS with substrate.

The following instance encompasses an entrenched FSS edifice. The component cell is a four-sided area placed among two 1-mm-thick dielectric sheets with permittivity ${ }_{r}{ }_{r}=2.0$, which is displayed as the supplement of Fig. 19. The power replication constant for standard frequency is displayed in Fig. 19. The outcome approves well with the MoM outcome by Mittra et al. [29]. 


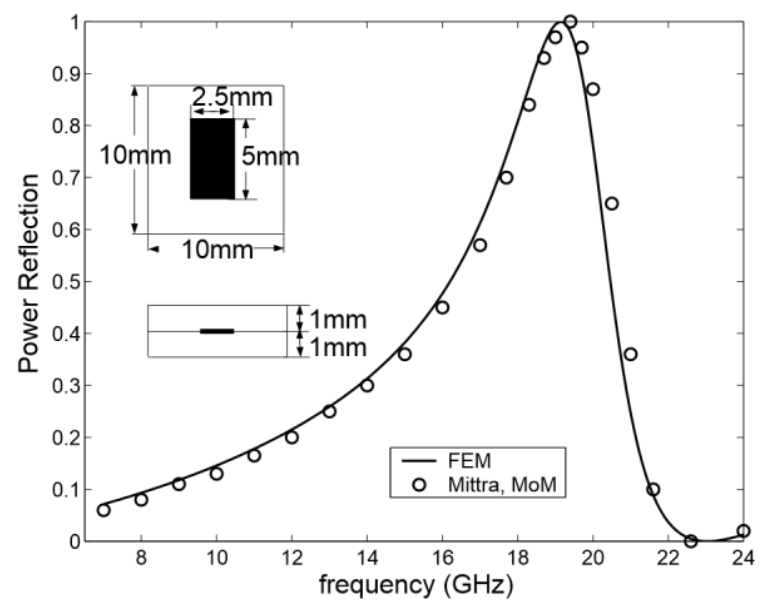

Fig. 19. Specular power replication constant intended for the entrenched area FSS.

In conclusion, we present a contrast amongst arithmetical outcomes and measured statistics. The edifice of this example is illustrated in Fig. 20(a). Two quadrilateral microstrip areas occur on the upper and lowest exteriors of an unchanging dielectric wedge with permittivity ${ }_{r}=2.5$. A PEC screen is located in the central of the slab and a quadrilateral slot is cut on the screen so that the two patches are coupled. The conduction constant for this FSS edifice has been dignified using the waveguide replication capacity technique in [31]. The contrast between the FEM outcome and the dignified statistics is displayed in Fig. 20(b). In the calculation, the episode viewpoint fluctuates bestowing to the dimension format defined in [31].

\subsection{Phased Selection Projections}

Phased selection projections are of countless significance in contemporary detector and communication methods. Modern combined aerial machinery has conveyed huge selections into extensive solicitations, and an upright empathy of such assortments usually needs arithmetical scrutiny. Precise estimate of selection constraints using arithmetical approaches not only diminishes enlargement expenses and project period but also reduces vital data to the project engineers. Existing arithmetical approaches comprise the MoM, the FEM, and more or less hybrid approaches [3], [7], [8], [35], [36].

In the midst of these approaches, the MoM has remained most widely employed for arithmetical study, and authentication has been made by likening with research outcomes for several

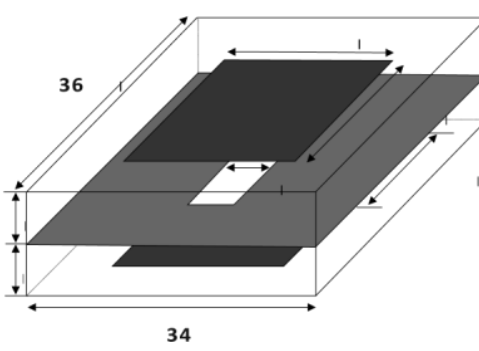

(a)

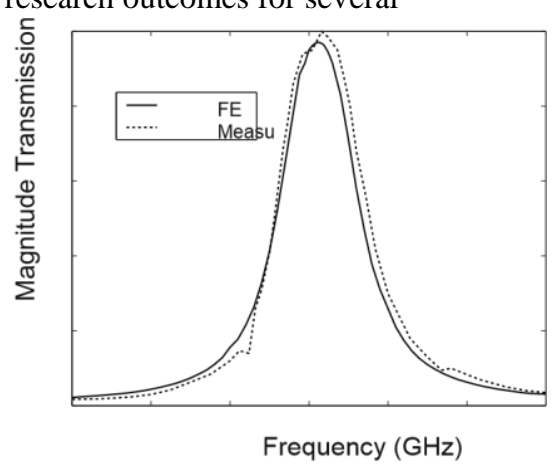

(b)

Fig. 20. (a) Geometry of the aperture-joined cover FSS. Dielectric filling is not displayed in the symbol. (b) Designed and dignified specular scale conduction constants.

projection structures. Cumulative stresses on selection enactments frequently need complex projects with inhomogeneous substantial configuration and unevenly molded conducting coverings. To correctly perfect the electromagnetic arena presentation in each selection component and the joint connection among the rudiments, a 3-D complete upsurge analysis is required. By way of an alternative characteristic of arithmetical molding, a precise study of radioactivity problems is incredible deprived of a cautious molding of the feeding edifices, which in itself can be complex for an effective project. In sight of these aims, the FEM seems to be most appropriate as an analysis instrument owing to its overall design and adaptability in geometry molding. 
This subdivision defines the use of the FEM for a 3-D radioactive analysis of immeasurable selection aerials. The FEM discretization is narrowed to a single component cell whose lateral exteriors and upper superficial are enacted with suitable episodic frontier conditions. The coaxial line feed is displayed in an exact way using the FEM web. On the coaxial port gap, a waveguide port condition is levied to shorten the web. The arithmetical outcomes gotten from this precise coaxial molding are likened with the outcomes acquired using the short existing investigation forage. For wideband calculations, the AWE method is used to hasten the incidence and angular curves. Arithmetical samples are assumed for microstrip-patch selections and wide notch projection displays.

\subsubsection{Feed Molding}

A significant arithmetical matter in the study of radioactivity problems is the appropriate modeling of the nurturing edifices. For the MoM, an exact demonstrating of the complete nurturing edifice is problematic, due to the 3-D nature of the nurturing edifices. In [35], an ideal current analysis was used to perfect the coaxial feed. As presented in Fig. 21(a), the basic probe-feed model comprises an ideal current thread through a tiny area and a tiny electrical dimension. A continuous current amplitude and period are expected on the strand. Even though the basic model envisages legitimately precise outcomes for micro strip selection projections with a tinny substrate and divulges very useful facts on image features, it does not take into justification the paraphernalia of the analysis range and the disparity of the current along the analysis, and thus is not relevant to microstrippatch selections with electrically copious substrates. On the extra hand, precise feed molding can be simply grasped using the FEM methods. By applying a very fine web to the forage area, one can precisely model the complete geometries of the nurturing edifices. For a coaxial forage, a steering analysis is shown as the allowance of the dominant electrode of the coaxial route, as shown in Fig. 21(b), and the PEC boundary condition is imposed on all the steering exteriors. On the coaxial space, the waveguide port state specified in Subdivision 2.5 is levied. Owing to the above method, we are capable of including the effects of the analysis range as well as the existing difference along the analysis. The fast arena difference around the coaxial-substrate connection area is also apprehended.

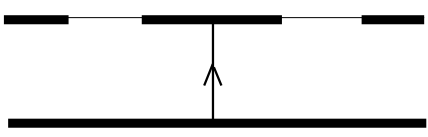

(a)

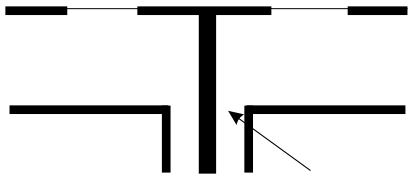

(b)

Fig. 21. Diagram of (a) tiny current analysis forage and (b) coaxial link forage.

Both basic and precise forage replicas can be simply applied in a FEM cipher. For the basic model, the basis current delivery $J_{s}$ is expected to be

$$
J_{s}=I_{0} \delta\left(x_{0}, y_{0}\right) \quad 0<z<h
$$

where $I_{0}$ is the current on the analysis which is placed at $\left(x_{0}, y_{0}\right)$ and outspreads from the earth surface up to an altitude $h$. As soon as the electric arena is achieved by resolving the structure matrix, the effort impedance is solved as

$$
\begin{aligned}
Z_{\text {in }} & =-\frac{1}{I_{0}^{2}} \int_{0}^{h} \mathbf{E} \cdot \hat{z} d z \\
& =-\frac{1}{I_{0}^{2}} \sum_{i}\left(\int_{0}^{h} \mathbf{N}_{i} \cdot \hat{z} d z\right) E_{i}
\end{aligned}
$$

where $i$ accounts for all the nonentities that are allied with the analysis

For the precise coaxial model, the replication back to the coaxial route is solved as the produce of the replicated arena with the overriding waveguide method $\mathrm{ZZ}$

$$
\begin{array}{rlr}
R & = & \mathbf{e}_{0} \cdot\left(\mathbf{E}-\mathbf{E}^{\mathrm{inc}}\right) d S \\
& \mathrm{X}_{S_{w}} \\
& =\underset{\substack{N_{i}{ }_{i}^{0} E_{i}-1 \\
i}}{ }
\end{array}
$$

where $S_{w}$ signifies the coaxial line space and $i$ accounts for all the nonentities on $S_{w}$. 


\subsubsection{Arithmetical Samples}

First, we present numerous microstrip-patch selection samples for the resolution of authentication since this kind of range has been carefully examined in the literature. Figure 22 displays the overall geometry of a microstrip area selection, in which metallic areas are positioned occasionally on a chastised dielectric substrate with altitude $h$ and permittivity ${ }_{r}$. The areas are nurtured with coaxial lines that go in the substrate from underneath the earth level. In order to model an immeasurable area selection on a substrate, the unit cell is usually properly chosen so that it contains only one area and the episodic frontier condition is levied on its four side exteriors. After the arenas are gotten in all places inside the component cell, preferred constraints such as effort impedance, vigorous replication or image constant, and dynamic component form can be simply calculated. The suitable description for the dynamic replication constant is specified by

$$
R(\theta, \phi)=\frac{Z_{i n}(\theta, \phi)-Z_{i n}(0,0)}{Z_{i n}(\theta, \phi)+Z_{i n}^{*}(0,0)}
$$

The use of the conjugate operative in (64) rebates the invented part of the effort impedance and extra evidently discloses the scan assets of the range.

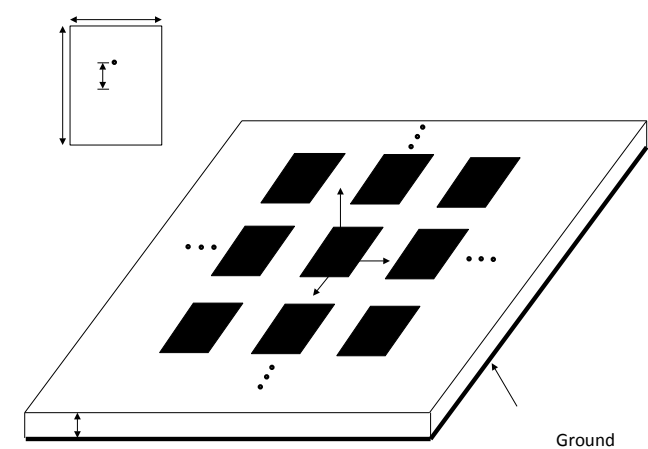

Fig. 22. Immeasurable microstrip area display on substrate.

Pozar and Schaubert [35] offered a comprehensive MoM study of microstrip area ranges which is used at this point as reference. The sample well-thought-out is a $0.3 \lambda_{0} \times 0.3 \lambda_{0}$ four-sided area selection with episodic distance $T_{x}=T_{y}=$ $0.5 \lambda_{0}$. The permittivity of the substrate is ${ }_{r}=2.55$. E-plane dynamic image constants are calculated for substrate altitude $h=0.02 \lambda_{0}$ and $0.06 \lambda_{0}$. The outcomes calculated using the basic and precise coaxial forage replicas are conspired in Fig. 23 unruffled with Pozar's MoM outcome, which used a basic analysis forage. It is well-known that all three outcomes come to an agreement with each other very well. All of them envisage an image impaired vision at $\theta=68.8^{\circ}$ for $h=$ $0.06 \lambda_{0}$, as an outcome of surface-wave excitation. Furthermore, the contract amid the basic and exact forage models proposes that image recitals such as dynamic replication constants are impervious to the precise forage system, as shown in [35].

The successive example is a microstrip range selection comprising of $0.25 \lambda_{0} \times 0.25 \lambda_{0}$ quadrilateral areas with episodic distance $T_{x}=T_{y}=0.5 \lambda_{0}$ and ${ }_{r}=2.5$. Figure 24 displays the contrast amid the effort impedances calculated with the basic analysis forage and precise coaxial line forage for two diverse substrate widths. It is well-known that the input resistances computed by the two replicas approve well once the substrate is thinner $\left(h=0.05 \lambda_{0}\right)$. In the instance of the denser substrate $\left(h=0.1 \lambda_{0}\right)$, the change amongst the two replicas is extra distinct, though the reverberating spectacle turn out to be less apparent. In both instances, the precise coaxial model envisages a somewhat minor reverberating incidence than the basic analysis model.

Subsequently, we contemplate a spherical area display nurtured with coaxial lines. The episodic distances are specified by $T_{x}=34 \mathrm{~mm}$ and $T_{y}=36.1 \mathrm{~mm}$, and the area
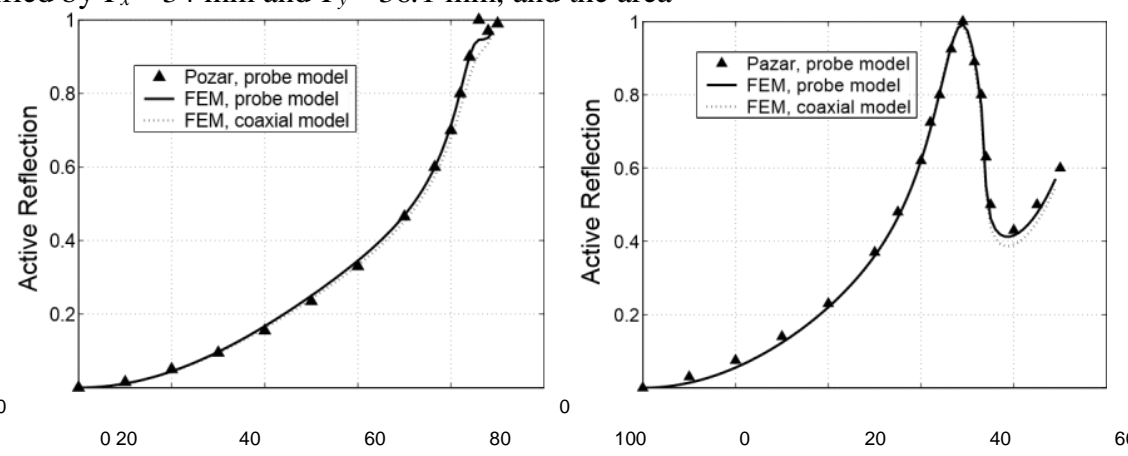

40

60

80

$100 \theta$ 
(a)

(degrees) $\theta$ (degrees)

(b)

Fig. 23. Dynamic replication constants for the microstrip area selection comprising of $0.3 \lambda_{0} \times 0.3 \lambda_{0}$ quadrilateral areas, $T_{x}=T_{y}=0.5 \lambda_{0},{ }_{r}{ }_{r}$ $=2.55$. (a) $h=0.02 \lambda_{0}, s=0.075 \lambda_{0}$; (b) $h=0.06 \lambda_{0}, s=0.14 \lambda_{0}$.

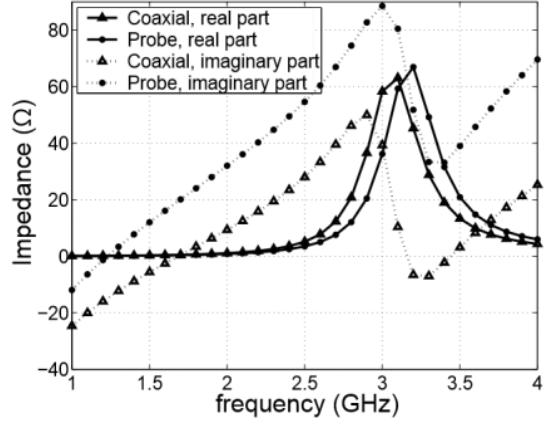

(a)

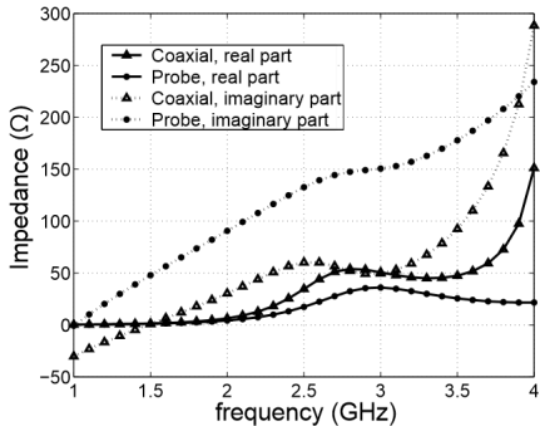

(b)

Fig. 24. Dynamic effort impedances for the microstrip area selection comprising of $0.25 \lambda_{0} \times 0.25 \lambda_{0}$ quadrilateral blotches, $T_{x}=T_{y}=$ $0.5 \lambda_{0}, s=0.0625 \lambda_{0},{ }_{r}=2.5$. (a) $h=0.05 \lambda ;$ (b) $h=0.1 \lambda$.

radius is $R_{0}=14.29 \mathrm{~mm}$. The external and internal ranges of the coaxial line used in the replication are $r_{o}=$ $1.492 \mathrm{~mm}$ and $r_{i}=0.456 \mathrm{~mm}$, correspondingly. The $S_{11}$ constraint for the coaxial port is displayed in Fig. 25 . Both the degree and segment of the calculated $S_{11}$ constraint reach agreement with the outcomes gotten by the cross sweeping scattering matrix (GSM) and the FEM [8]. In the FEM estimation, the event viewpoint differs bestowing to the waveguide simulant dimension in [8].

The widening indentation selection aerial, or the Vivaldi range antenna, is recognized as an upright aspirant for wide-band and wide-scan projections. Figure 26

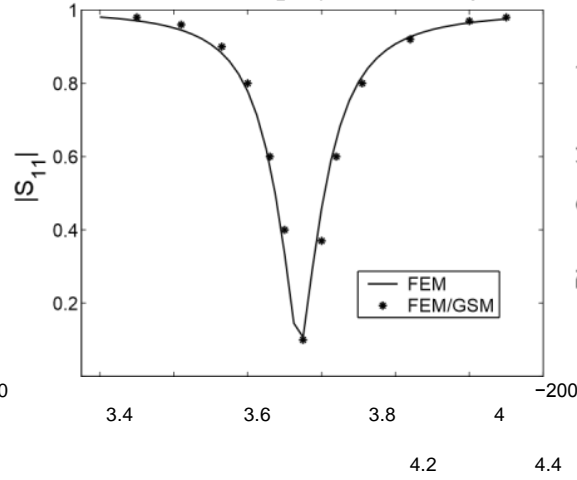

(a)

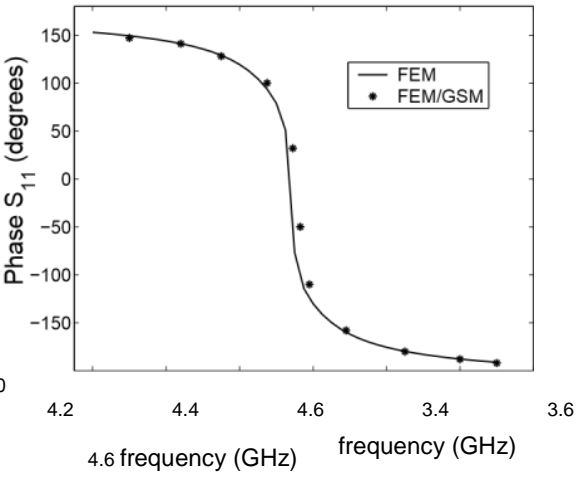

(b)

Fig. 25. (a) Degree and (b) segment of the $S_{11}$ constraint for the microstrip area selection comprising of spherical areas with radius $R_{0}=$ $14.29 \mathrm{~mm}, h=0.79 \mathrm{~mm},{ }_{r}{ }_{r}=2.33$. Forage is molded using a accurate coaxial line model with $r_{o}=1.492 \mathrm{~mm}, r_{i}=0.456 \mathrm{~mm}$. Forage offset is $s=5 \mathrm{~mm}$, and permittivity of the coaxial line is ${ }^{2}=2.024$.

displays the component cell geometry of a widening indentation selection aerial. A piloting area is made on one lateral of a dielectric wedge, which attitudes sheer above a milled level. A thin slit streak is cut in the central of the steering area and is steadily widening into an open aperture to offer a level impedance change from the nurturing system to the permitted interplanetary.

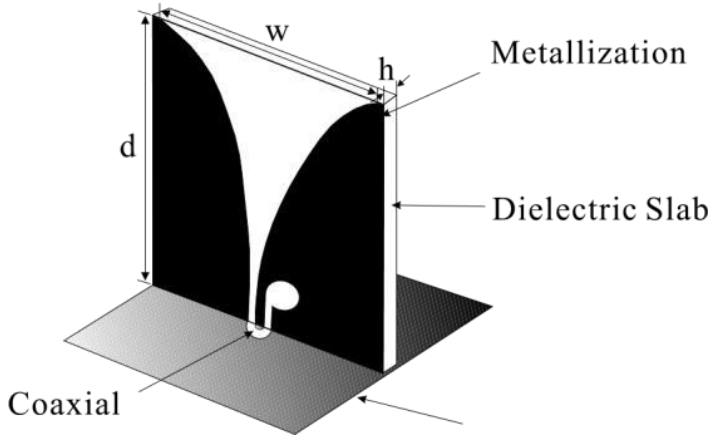


Fig. 26. Component cell of the widening indentation projection array.

According to Sakyi K.S. et al, the projection porotype and constraints used in our estimation keep an eye on a project defined in [7]. The probe is constructed for single-polarized procedures and is fed by a small unit of a coplanar waveguide that links the well-adjusted coaxial route and the unstable slot line. The coplanar waveguide comprises of two arms: one develops as the wide slot line and the other finishes at a deep loop which functions as a wide-band sweeping circuit. The coplanar waveguide is formerly linked to a coaxial line by joining their dominant electrodes. The benefit of this project is that metallization is useful to only a single side of the dielectric lump and no via-holes are required for ground networks. The selection space in the $x$ and $y$ axis is $T_{x}$ and $T_{y}$, correspondingly. The altitude and width of the dielectric lump are 33.3 and $1.27 \mathrm{~mm}$. The partial thickness of the slot line varies with $z$ rendering to an exponential function specified by

$$
w(z)=0.25 e^{0.123 z}
$$

According to Koram Samuel (2018), this function provides a partial breadth of $15 \mathrm{~mm}$ at the sweeping aperture. The resonating sphere has a radius of $2.5 \mathrm{~mm}$. The coaxial line is molded as a space censored on the ground level with its internal and external radius being 0.375 and $0.875 \mathrm{~mm}$, correspondingly. Figure 27 displays the dynamic replication constant as a function of incidence at broadside image viewpoint. The three curvatures agree to $T_{x}=32,36$, and $40 \mathrm{~mm}$, correspondingly. In all these instances, $T_{y}$ is secured to be $34 \mathrm{~mm}$. It is established that a severe replication height acts in all three curvatures and the resultant incidence of the top upsurges as the episodic distance in the $x$ axis is reduced. A related comportment has been detected earlier in the image recitals of one- and two polarized elongated slit projection ranges with double-printed substrate, which is stated as impedance differences [37]. These impedance differences have been created to transmit to the significances of the void made by the substrate area. Here we service similar void model, with the 2-D cavity demarcated by the two end-to-end rows of blistering areas and the ground level. By presumptuous the PEC settings on the dual side walls and the lowest superficial and the PMC form on the highest superficial, one can simply calculate the booming incidences of the 2-D cavity. The central method is the $T E_{11}$ approach and the resultant booming incidence is specified by

$$
f_{r}=\frac{c_{o}}{2} \sqrt{\left(\frac{m}{T_{x}}\right)^{2}+\left(\frac{2 p+1}{2 h}\right)^{2}}
$$

where $T_{x}$ is the episodic distance in the $x$ axis and $h$ is the altitude of the substrate. For the three diverse standards of $T_{x}$ in Fig. 27, the estimated quality incidences are 4.30, 4.73, and 5.19 GHz, which are near to the replication points $(4.24,4.6$, and $5.08 \mathrm{GHz})$ in Fig. 27. It is likewise perceived that the quality incidence does not differ when the image path is directed off broadside, further signifying that the occurrence is allied with certain geometrical constraints of the component cell itself.

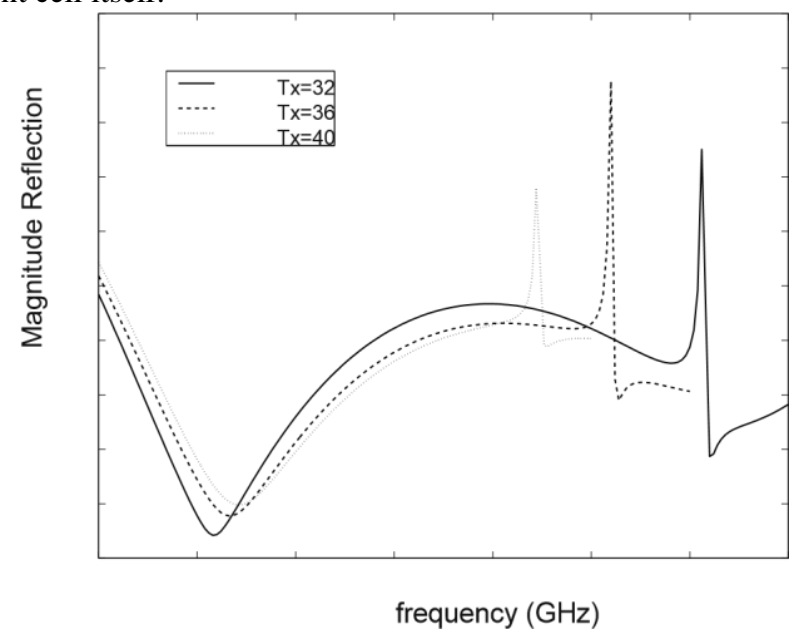

Fig. 27. Dynamic replication constant for the wide nick projection range with diverse selection space in the $x$ axis.

Subsequently, the image recitals of the wide nick range are calculated. Figure 28 displays the E-plane and H-plane dynamic replication constants at $f=2.5,3.0$, and $4.8 \mathrm{GHz}$. The figure shows that effort impedance vicissitudes easily 
with image viewpoint over a varied angular array at both 2.5 and $3.0 \mathrm{GHz}$. At $4.8 \mathrm{GHz}$, where the range space becomes bigger than one-half wavelength, the discordant effect-induced image blindness acts in both E-plane $\left(\theta=57^{\circ}\right)$ and $\mathrm{H}$ plane $\left(\theta=47^{\circ}\right)$ images. In the E-plane, there is an extra loss of sight at $\theta=17^{\circ}$, which reach agreement with the outcome by McGrath [7]. Some scholars have elucidated this occurrence as the excitation of assured waveguide methods on a wavy edifice [38].

\section{Conclusions}

This chapter defines an FEM exploration of enormously episodic edifices. The hypothetical root for the exploration is the central FEM theory joined with the Floquet theorem and the central reckoning invention. Higher-order trajectory base functions are employed to enlarge electromagnetic arenas. Arithmetical outcomes validate the efficacy and accurateness of using advanced order trajectory base functions. The AWE-based method is joined

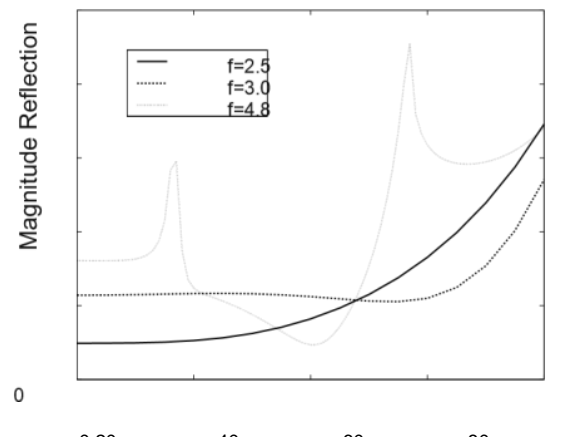

(a)

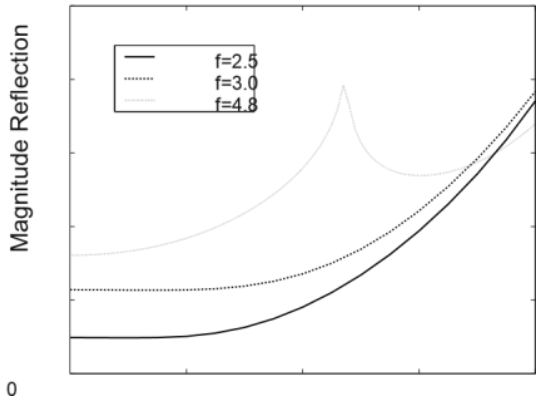

20

(b)

Fig. 28. Dynamic replication constant for the wide nick projection array with $T_{x}=36 \mathrm{~mm}$ at $f=2.5,3.0$, and $4.6 \mathrm{GHz}$. (a) E-plane image. (b) H-plane image.

with FEM to implement wild incidence and angular curves. The FEM forage molding is also deliberated in point for radioactive problems. The legitimacy and adaptability of the FEM design have been established through several arithmetical samples offered in Section 2. Most of the samples are offered for authentication purposes, over which contrast with earlier distributed outcomes can be made. More refined projects are not enclosed in this episode, nonetheless they can be evaluated simply by the same technique industrialized here owing to the elastic volumetric molding of the FEM.

\section{Acknowledgments}

This work was financially supported by Mr. Samuel Sakyi Koram a PhD in Solid Mechanics student with the Department of Mechanics and Engineering Science, School of Civil Engineering and Mechanics - Jiangsu University, China. Moreover I'd like to express my heartfelt gratitude to my current supervisor in the person of Professor Jian-Fei Lu of the Faculty of Civil Engineering and Mechanics, Jiangsu University, Zhenjiang- China for his remarkable, noteworthy contributions and advice to make this a success.

\section{References}

1. S. Gedney and R. Mittra, "Analysis of the electromagnetic scattering by thick gratings using a combined FEM/MM solution," IEEE Trans. Antennas Propagat., vol. 39, pp. 1605-1614, Nov. 1991.

2. J. M. Jin and J. L. Volakis, "A hybrid finite element method for scattering and radiation by microstrip patch antennas and arrays residing in a cavity," IEEE Trans. Antennas Propagat., vol. 39, pp. 1598-1604, Nov. 1991.

3. E. W. Lucas and T. P. Fontana, "A 3-D hybrid finite element/boundary element method for the unified radiation and scattering analysis of general infinite periodic arrays," IEEE Trans. Antennas Propagat., vol. 43, pp. 145-153, Feb. 1995.

4. J. L. Volakis, T. F. Eibert, D. S. Filipovic, Y. E. Erdemli, and E. Topsakal, "Hybrid finite element methods for array and fss analysis using multiresolution elements and fast integral techniques," Electromagn., vol. 22, pp. 297-313, MayJun 2002.

5. Sakyi, K.S. and Lu, J.F., "Application of the Laplace transform to non-rectilinear momentary problems." Journal of Innovative Systems Design and Engineering. Pages 29 to 38.

6. K. Samuel, B. Korankye, and G. Kweitsu, "Seismic response analysis of underground structures", J. Enviro. Earth Sci, vol. 8, no. 5, pp. 48-63, 2018. 
7. J. M. Jin and J. L. Volakis, "Scattering and radiation analysis of threedimensional cavity array via a hybrid finiteelement method," IEEE Trans. Antennas Propagat., vol. 41, pp. 1580-1586, Nov. 1993.

8. D. T. McGrath and V. P. Pyati, "Phased array antenna analysis with the hybrid finite element method," IEEE Trans. Antennas Propagat., vol. 42, pp. 1625-1630, Dec. 1994.

9. M. A. Gonzalez, J. A. Encinar, J. Zapata, and M. Lambea, "Full-wave analysis of cavity-backed and probe-fed microstrip patch arrays by a hybrid modematching generalized scattering matrix and finite-element method," IEEE Trans. Antennas Propagat., vol. 46, pp. 1998, Feb. 1998.

10. J. M. Jin, The Finite Element Method in Electromagnetics, Wiley, New York, 2nd ed., 2002.

11. R. D. Graglia, D. R. Wilton, and A. F. Peterson, "Higher order interpolatory vector bases for computational electromagnetics," IEEE Trans. Antennas Propagat., vol. 45, pp. 329-340, Mar. 1997.

12. I. S. Duff, A. M. Erisman, and J. K. Reid, Direct Methods for Sparse Matrices, Clarendon Press, Oxford, 1986.

13. L. T. Pillage and R. A. Rohrer, "Asymptotic waveform evaluation for timing analysis," IEEE Trans. Comput.-Aided Des., vol. CAD-9, pp. 352-366, April 1990.

14. Q. J. Zhang, M. A. Kolbehdari, M. S. Nakhla, and R. Achar, "Simultaneous time and frequency domain solutions of em problems using finite element and cfh techniques," IEEE Trans. Microwave Theory Tech., vol. 44, pp. 1526- 1534, Nov. 1993.

15. M. Li, Q. J. Zhang, and M. S. Nakhla, "Finite difference solution of em fields by asymptotic waveform techniques," IEE Proc. H, vol. 143, pp. 512-520, Dec. 1996.

16. C. J. Reddy, M. D. Deshpande, C. R. Cockrell, and F. B. Beck, "Fast RCS computation over a frequency band using method of moments in conjunction with asymptotic waveform evaluation technique," IEEE Trans. Antennas Propagat., vol. 46, pp. 1229-1233, Aug. 1998.

17. J. Liu, J. M. Jin, E. K. N. Yung, and R. S. Chen, "A fast three-dimensional higher-order finite element analysis of microwave waveguide devices," Microwave Opt. Tech. Lett., vol. 32, pp. 344-352, Mar. 2002.

18. R. T. Tiberio, G. Pelosi, and G. Manara, "A uniform gtd formulation for the diffraction by a wedge with impedance faces," IEEE Trans. Antennas Propagat., vol. 33, pp. 867-873, Aug. 1985.

19. E. F. Kuester and C. L. Holloway, "A low-frequency model for wedge or pyramid absorber arrays-I: theory," IEEE Trans. Electromagn. Compat., vol. 36, pp. 300-306, Nov. 1994.

20. E. F. Kuester and C. L. Holloway, "A low-frequency model for wedge or pyramid absorber arrays-II: computed and measured results,” IEEE Trans. Electromagn. Compat., vol. 36, pp. 307-313, Nov. 1994.

21. R. Janaswamy, "Oblique scattering from lossy periodic surfaces with application to anechoic chamber absorbers," IEEE Trans. Antennas Propagat., vol. 40, pp. 162-169, Feb. 1992.

22. N. Marly, D. D. Zutter, and H. Pues, "A surface integral equation approach to the scattering and absorption of doubly periodic lossy structures,” IEEE Trans. Electromagn. Compat., vol. 36, pp. 14-22, Feb. 1994.

23. N. Marly, D. D. Zutter, and H. Pues, "Integral equation modeling of the scattering and absorption of multilayered doublyperiodic lossy structures," IEEE Trans. Antennas Propagat., vol. 43, pp. 1281-1287, Nov. 1995.

24. C. F. Yang, W. D. Burnside, and R. C. Rudduck, "A doubly periodic moment method solution for the analysis and design of an absorber covered wall," IEEE Trans. Antennas Propagat., vol. 41, pp. 600-609, May 1993.

25. W. Sun, K. Liu, and C. A. Balanis, "Analysis of single and doubly periodic absorbers by frequency-domain finitedifference method," IEEE Trans. Antennas Propagat., vol. 44, pp. 798-805, June 1996.

26. K. Liu, W. Sun, and C. A. Balanis, "A periodic finite-difference method for microwave absorber analysis and design," 1994 IEEE Antennas Propagat. Soc. Int. Symp., vol. 3, pp. 1400-1403, 1994.

27. C. L. Holloway, P. M. McKenna, R. A. Dalke, R. A. Perala, and C. L. Devor, "Time-domain modeling, characterization, and measurements of anechoic and semi-anechoic electromagnetic test chambers," IEEE Trans. Electromagn. Compat., vol. 44, pp. 102-118, Feb. 2002.

28. Y. C. Chung, B. W. Kim, and D. C. Park, "Range of validity of transmission line approximations for design of electromagnetic absorbers," IEEE Trans. Magn., vol. 33, pp. 1188-1192, July 2000.

29. H. L. Bertoni, L.-H. S. Cheo, and T. Tamir, "Frequency-selective reflection and transmission by a periodic dielectric layer," IEEE Trans. Antennas Propagat., vol. 37, pp. 78-83, Jan. 1989.

30. R. Mittra, C. H. Chan, and T. Cwik, "Techniques for analyzing frequency selective surfaces—a review," Proc. IEEE, vol. 76, pp. 1593-1610, Dec. 1988.

31. J. M. Jin and J. L. Volakis, "Electromagnetic scattering by a perfectly conducting patch array on a dielectric slab," IEEE Trans. Antennas Propagat., vol. 38, pp. 556-563, Apr. 1990.

32. R. Pous and D. M. Pozar, "A frequency-selective surface using aperturecoupled microstrip patches," IEEE Trans. Antennas Propagat., vol. 33, pp. 653-678, May 1998.

33. J. Vazquez, C. G. Parini, and P. J. B. Clarricoats, "The finite difference modelling of infinite arrays in time domain," National Conference on Antenna and Propagation, pp. 209-212, Aug. 1999.

34. D. M. Pozar and D. H. Schaubert, "Analysis of an infinite array of rectangular microstrip patches with idealized probe feeds,” IEEE Trans. Antennas Propagat., vol. 32, pp. 1101-1107, Oct. 1984. 
35. C.-C. Liu, A. Hessel, and J. Shmoys, "Performance of probe-fed microstrippatch element phased arrays," IEEE Trans. Antennas Propagat., vol. 36, pp. 1501-1509, Nov. 1988.

36. T.-H. Chio H. Holter and D. H. Schaubert, "Elimination of impedance anomalies in single- and dual-polarized endfire tapered slot phased arrays," IEEE Trans. Antennas Propagat., vol. 48, pp. 122-124, Feb 2000.

37. D. H. Schaubert and J. A. Aas, "An explanation of some e-plane scan blindnesses in single-polarized tapered slot arrays," IEEE APS Int. Symp. Dig., vol. 33, pp. 1612-1615, June 1993. 